\title{
COVID-19: Attacks the 1-beta Chain of Hemoglobin to Disrupt Respiratory Function and Escape Immunity
}

\author{
Liu Wenzhong ${ }^{1,2, *}$, Li Hualan ${ }^{2}$ \\ 1 School of Computer Science and Engineering, Sichuan University of Science \& Engineering, \\ Zigong, 643002, China; \\ 2 School of Life Science and Food Engineering, Yibin University, Yibin, 644000, China; \\ ${ }^{*}$ Correspondence.Wenzhong Liu, liuwz@suse.edu.cn.
}

\begin{abstract}
Investigating poor respiratory function and high immunological escape in COVID-19 patients may aid in the prevention of additional deaths. The conserved domain search method was used to evaluate the biological roles of specific SARS-COV-2 proteins in this present study. The research findings indicate that the SARS-COV-2 virus contains domains capable of binding porphyrin and synthesizing heme. $\mathrm{S}$ and ORF3a can bind to hemoglobin. The $\mathrm{S}$ protein possesses hemocyanin-like function since it contains copper-oxygen binding, immunological agglutination, and phenoloxidase domains. ORF3a's Arg134 and E's Cys44 have heme-iron binding sites, respectively. The ORF3a protein has a region that degrades trapped heme into iron and porphyrin. Hemoglobin that has been attacked by ORF3a may preserve the majority of its native structure but with decreased oxygen delivery function. By targeting hemoglobin and destroying heme, the ORF3a protein caused varying degrees of respiratory distress and coagulation symptoms in COVID-19 individuals. ORF3a of Delta and Omicron variants also retained its capacity to target hemoglobin and heme. But the S protein's hemocyanin-like domain transported oxygen to enhance the patient's respiratory condition. Through a large load of hemocyanin-like proteins, the mutant virus achieved effective oxygen transport and alleviated the symptoms of respiratory distress in patients. Simultaneously, the variant S protein's immunological agglutination and phenol oxidase functions were decreased or eliminated, resulting in a decrease in the strength of the immune response and an increase in immune evasion ability, culminating in increased virus transmission.
\end{abstract}

Keywords: Porphyrin; Heme; Hemocyanin; Immunological agglutination; Phenoloxidase; Melanin; Oxyhemoglobin Dissociation curve; Humoral immune response; Happy hypoxia; Delta Variant; Omicron Variant;

\section{Background}

COVID-19 patients exhibit symptoms such as diarrhea(1, 2), hypotension(3), and electrolyte abnormalities(4). Early asymptomatic infections cause taste and smell $\operatorname{loss}(5,6)$. Patients with severe COVID-19 also have a higher risk of developing rare neurological illnesses such as epilepsy $(7,8)$ and encephalitis $(9,10)$. Cytokine storm causes organ failure in the later stages of severe inflammatory infection, including the heart(11), liver(12), and kidneys(13). Patients with COVID-19 will suffer varying respiratory distress symptoms during the early and severe stages. Numerous ground-glass pictures and piercing shadows occur on both sides of the lungs $(14,15)$. Ground-glass images are frequently linked with severe hypoxia. Despite significant hypoxemia, some individuals with COVID-19 pneumonia experience no dyspnea, demonstrating the "happy 
hypoxia" paradox. Severe patients rescued with Extracorporeal Membrane Oxygenation (ECMO) exhibit unusual clinical characteristics, including low oxygen, low blood oxygen saturation(16, 17), and high dissolved oxygen levels. Thus, elucidating the mystery respiratory issues and immune response of COVID-19 patients may be a critical step for increasing patient rescue success rates(18).

Colistin B and coronavirus may result in significant skin darkening in patients with COVID-19 who are rescued with ECMO(19). Dermatological symptoms of COVID-19 include(20): urticaria, erythema confluent/maculopapular/measles-like $\operatorname{rash}(21)$, papular rash, chilblain-like lesions, livedo reticularis/racemoid pattern, and purpura" Vasculitis". The erythema associated with certain severe skin diseases might be converted to hyperpigmentation. Patients with COVID-19 ocular infection develop unilateral acute posterior multifocal lamellar pigment epitheliopathy(22). COVID-19 patients have bilateral pigmentation of the corneal endothelium, pigment dispersion in the anterior chamber, iris depigmentation with iris transillumination abnormalities(23). Melanin also has a vital role in the coloration of the oral mucosa(24). Oral hyperpigmentation caused by HIV can affect any area of the oral mucosa(25). Melanin affects the inflammatory response directly or indirectly by influencing the generation of host cytokines/chemokines(26). Melanin alters the signaling cascades mediated by cytokines. It boosts the release of pro-inflammatory mediators such as interleukin (IL)-1, IL-6, interferon gamma, and tumor necrosis factor(26).

Living creatures develop pigments to protect themselves from UV rays. Melanin is the most strong and efficient of all pigments. When UV radiation strikes melanin's chemical chain, the chemical chain vibrates at an incredibly high rate, turning dangerous UV light into innocuous heat. When the retina is activated by damaging light, harmful free radicals are created, initiating a sequence of destructive oxidative events on the retina. Melanin can act as an antioxidant and protect the eyes by neutralizing these damaging oxidation events. Melanin also protects microorganisms from enzymatic destruction, radiation (UV, sunlight, gamma), and heavy metals, as well as increasing their thermal tolerance (to heat and cold)(27). Melanin enhances virulence by shielding fungal cells from phagocytosis by immune effector cells(28). Bacterial melanin-like pigments have been shown to be capable of scavenging superoxide anion free radicals and inhibiting monocytes' respiratory burst response(29). Through melanin, the SARs-COV-2 virus may achieve immune evasion and radiation protection. However, in COVID-19 patients, an overabundance of synthetic melanin might result in hyperpigmentation.

Pigmentation also occurs in some pathogen-infected lower species. Hemocyanin's extracellular phenoloxidase (PO) is the primary source of hyperpigmentation (melanosis) in shellfish(30, 31). Phenoloxidase activity resulting from hemocyanins contributes to brown algal hyperpigmentation. Hemocyanin is the pigment that causes hyperpigmentation in $N$. norvegicus (32). Hemocyanin is homologous to phenoloxidases such as tyrosinase because both proteins have a type $3 \mathrm{Cu}$ active site coordination(33). Enzymes of phenoloxidase (PO), such as tyrosinase (EC 1.14.18.1) and catechol oxidase (EC 1.10.3.1). Catecholamine (CA) is a catechol derivative. Epinephrine (E), norepinephrine (NE), and dopamine (D) are all endogenous CA. The enzymatic reaction catalyzed by phenoloxidase results in chromogen, melanin, and other pigments(34). The phenoloxidase (PO) enzymes significantly contribute to hyperpigmentation(35). Insect prophenoloxidase (PPO) is also an important innate immune protein as it is involved in cellular and humoral defenses(36). 
Hemocyanin comprises three domains(37): a $\mathrm{N}$-terminal domain, an active site containing binuclear copper ions, and a C-terminal domain(38). Conformational changes in the $\mathrm{N}$-terminal domain can activate hemocyanin's phenoloxidase activity. The C-terminal domain contribute in organisms' immunological agglutination activity(39) and increase blood cell phagocytosis(40). Hemocyanin is found only in hemolymph and appears in hexamers or hexameric oligomers(41). The active site catalyzes the chelation of two copper ions and the binding of an oxygen molecule (42). Hemocyanin is more than twice the size of hemoglobin(43). Hemocyanin reversibly binds to oxygen molecules via altering the valence of copper ions $\left(\mathrm{Cu}^{2}+\leftrightarrow \mathrm{Cu}+\right)$ (44). It binds 96 oxygen molecules, whereas hemoglobin only binds four(43).

Hemocyanin molecules float freely in the blood, whereas red blood cells contain millions of smaller hemoglobin molecules(43). Hemocyanin and hemoglobin have complementary distributions in some insect orders(45) and crustaceans(46). Hemocyanin and hemoglobin are both present in the crustaceans, Hymenoptera and Hemiptera. Hemocyanin works as a physiological supplement, compensating for poor oxygen transport in the trachea and assisting insect embryos in aerobic respiration(47). Hemocyanin expression was dramatically increased in Baifutiao(48) and locust embryos(49) under hypoxia conditions compared to normoxic settings. Increased hemocyanin content assists locust embryos in obtaining adequate and steady oxygen in hypoxic high altitude locations(49). Hemocyanin expression was also significantly elevated in the blue crab Callinectes sapidus (50) and the ecliptic crab Cancer magister(51) under hypoxic conditions (52).

Altitude sickness' physiological characteristics and symptoms at high altitude are comparable to those of various illnesses related to COVID-19(53). The SARs-COV-2 virus may contain a hemocyanin-like protein that binds oxygen and activates the phenoloxidase activity of the virus. The phenoloxidase structure synthesizes melanin. COVID-19 patients had hemoglobin and hemocyanin-like co-transport oxygen patterns. Hemocyanin-mediated oxygenation leads to effective $\mathrm{O} 2$ transport under hypoxic settings(54). In this mode, oxygen supplied by hemocyanin-like compensates for bodily hypoxia even when red blood cells or hemoglobin were not working adequately. However, the current approach of light absorption in oxygenation detection considered hemoglobin solely and ignored oxygen-carrying hemocyanin-like. Present blood gas detection technologies presupposed that the oxygen molecules in the blood were dissolved oxygen which was not bound to hemoglobin(55). But the implicit condition that hemocyanin-like could also bind oxygen molecules was ignored. $\mathrm{pO}_{2}$ determined how much oxygen was bound by hemoglobin and hemocyanin-like proteins. Existing assays overestimated hemoglobin's oxygen supply status. The patient's oxygenation curve may remain unchanged. The actual oxygenation curve should be moved to the right, lowering hemoglobin's affinity for oxygen. Thus, when viruses attacked hemoglobin, it exhibited "high dissolved oxygen". In contrast, hemocyanin-like proteins exhibited an abnormal oxygen transport function, resulting in "happy hypoxia."

To collect iron and heme from host proteins like hemoglobin in red blood cells, pathogens have evolved various iron absorption methods(56). According to a computational research, the SARs-COV-2 virus infects erythrocytes via the Plasmodium falciparum-like and complement-like domains. Anupam Mitra discovered a viral leukocyte response in COVID-19-infected patients(57). According to Bhardwaj, $\mathrm{pRb}$ and its interaction with Nsp15 affect coronavirus infection(58). Electron microscopy revealed the presence of the Colorado tick fever virus in red blood cells(59). 
Plasmodium, Babesia, and trypanosomes also infect red blood cells, eliciting symptoms similar to COVID-19. Trypanosome extracellular vesicles fuses with mammalian erythrocytes(60), resulting in simple erythrocyte removal and additional anemia. Babesia invasion of red blood cells requires adhesion proteins and apical membrane antigens associated with thrombin sensitivity(61).

Pathogenic E. coli strains can use hemoglobin as a source of iron(62). Hemoglobin protease $(\mathrm{Hbp})$ is a proteolytic enzyme similar to IgA1 protease. Immunoglobulin A1 protease (IgA1 protease) is a serine protease (S6 family) that is produced by several pathogenic bacteria(63), including those that cause bacterial meningitis, Haemophilus influenzae, Neisseria meningitidis, and Chain pneumonia Cocci(63). The IgA1 protease colonizes human mucosal surfaces, affecting specific immune responses(63). This serine protease autotransporter, released from E.coli, destroys hemoglobin, binds the liberated heme, and transports it to both bacteria(56).

When Staphylococcus aureus's Isd protein degrades hemoglobin, the 1-beta chain is targeted first, releasing heme and initiating a series of heme release events. Heme transfer from met- $\mathrm{Hb}$ to $\mathrm{IsdH} / \mathrm{B}$ is slower than from met- $\mathrm{Hb}$ to full-length $\mathrm{Hb}$ receptors(64). It is also governed by simple heme dissociation from met- $\mathrm{Hb}(65)$. By binding to $\alpha \mathrm{Hb}$ via the IsdH or IsdB domains, the rate of efficient interactions between the $\mathrm{Hb}$ chain and the heme receptor domain is increased(66). This binding is used to specifically target the heme receptor domain, regulating the sequential release of heme from $\beta \mathrm{Hb}$ and $\alpha \mathrm{Hb}$ chains(66). The $\alpha \mathrm{Hb} \cdot \beta \mathrm{Hb}$ dimer releases heme from a single subunit (half- $\mathrm{Hb}$ ) while retaining the majority of its natural structure(67). It remains linked with the $\mathrm{Hb}$ receptor until all heme is released. IsdH does not bind to free apo-Hb. The IsdH-Hb complex dissociates only when the heme in $\mathrm{Hb}$ is completely removed(66). The SARs-COV-2 viral protein may interact with $\alpha \mathrm{Hb}$ via the Isd domains and then break $\beta \mathrm{Hb}$ using the IgA1 protease structure. This attack on the 1-beta chain of hemoglobin initiated the sequential release of heme from hemoglobin. The IsdC protein from $S$. aureus uses a flexible binding pocket to capture heme(68). The crystal structure of the heme-IsdC complex is the central conduit of the $S$. aureus Isd iron/heme uptake system(69). IsdA, IsdB, IsdH, and IsdC share the same heme-binding module, termed the NEAT (near transporter) domain(69). But the iron-regulated surface proteins IsdA, $\mathrm{IsdB}$, and IsdH may be not required for heme iron utilization in $S$. aureus (70).

Heme is a kind of metalloporphyrin. Heme activates signaling pathways independent of iron and reactive oxygen species (ROS), including those involved in redox metabolism(71). Heme metabolism is required for Plasmodium, the causative agent of malaria, to infect red blood cells(72). Numerous Plasmodium genes encoding heme-binding proteins have been found(73). Hemozoin is frequently referred to as malaria pigment in malaria parasites. Phagocytosis for the malaria pigment heme inhibits CD54 and CD11c expression in human monocytes(59). Hemozoin (malarial pigment) impairs the differentiation and maturation of dendritic cells generated from human monocytes(57). In vitro, the malaria-specific metabolite hemozoin promotes the production of three powerful endogenous pyrogens (TNF, MIP-1, and MIP-1), while in vivo, it changes thermoregulation(58). Hemozoin is involved in various processes that may contribute to Plasmodium pathogenicity. Numerous antimalarial medicines, including chloroquine, act by selectively inhibiting this hemozoin detoxification/hemozoin production pathway(74).

Iron is most frequently liberated from heme's oxidative breakdown by heme oxygenase (HO) Most of the time, iron is released from heme due to the protoporphyrin ring degrading(75). $C$. diphtheriae's heme oxygenase degrades heme to generate $\alpha$-biliverdin, carbon monoxide, and free iron(76). IsdG and IsdI (heme oxygenase) of Staphylococcus aureus cleaves the tetrapyrrole ring 
structure of heme in the presence of NADPH cytochrome P450 reductase, thereby releasing iron.(77). However, E.coli's deferrochelating activity does not destroy the tetrapyrrole backbone(78). It is the case with Yersinia enterocolitis HemS(79) and E. coli $\mathrm{O} 157: \mathrm{H} 7 \mathrm{ChuS}(80)$. HemS is used by E. coli Bartonella hensii to deal with oxidative stress caused by $\mathrm{H}_{2} \mathrm{O}_{2}(81)$. Iron is released from heme by the HemS of E. coli Bartonella henii without causing damage to the tetrapyrrole backbone(81). HemS protein also degrades heme in the presence of electron donors, ascorbate, or NADPH-cytochrome $\mathrm{P} 450$ reductase(81).

HemS protein stimulates the release of iron from heme, leaving behind hematoporphyrin. Most porphyrin molecules are hydrophobic and agglomerate in water(82). Porphyrin photosensitizers with a higher hydrophobicity penetrate mammalian cell membranes(83). Porphyrins diffuse through the phospholipid bilayer and accumulate in the cytoplasm due to concentration gradients(84). Porphyrin chemicals, such as synthetic photosensitizers, are frequently utilized to treat cancers by photodynamic therapy(85). Porphyrin derivatives get in cancer cells through endocytosis and concentration gradient osmosis(86). Porphyrins produce reactive oxygen species (ROS) that kill tumor cells(87). The SARS-CoV-2 viral proteins bound porphyrin (or heme) to get energy and cell membrane penetration. It generated reactive oxygen species (ROS) to damage the cell membrane. Porphyrins are mostly preserved in the human body as heme on hemoglobin. The virus's high requirement for porphyrin and iron developed to attack hemoglobin and broke heme into iron and porphyrin.

The disorder of the body's porphyrin metabolism causes Porphyria. Atypical porphyrins have been identified in patients with acquired immunodeficiency syndrome(88). Chronic hepatic porphyria, not delayed cutaneous porphyria, is the form of porphyrin metabolic illness caused by the hepatitis C virus(88). Numerous clinical observations have revealed that patients with COVID-19 also have skin and nervous system symptoms consistent with porphyria. Genetic abnormalities in the heme biosynthesis enzyme uroporphyrinogen III synthase also cause congenital erythropoietic porphyria(89). The SARs-COV-2 viral protein may has uroporphyrinogen III synthase activity and contribute to infection by producing comparable heme. This type of uroporphyrinogen III synthase inhibited the metabolism of porphyrins.

We employed the conserved domain search method to study the SARs-COV-2 viral proteins in this work. The results indicated that the SARS-COV-2 virus could synthesize heme from porphobilinogen and encodes all necessary enzymes for this process. The SARs-COV-2 ORF3a protein could target 1-beta chain hemoglobin and dissociate heme to iron and porphyrin. These attacks by the SARs-COV-2 virus and its variants could cause severe damage to respiratory tissues and organs. Meanwhile, the SARs-COV-2 virus's S protein exhibited hemocyanin-like activity. The S protein's hemocyanin-like domain transported oxygen to enhance the patient's respiratory condition and affect immune response.

\section{Methods}

\subsection{Data set}

1. The sequences of SARS-COV-2 proteins. The SARS-COV-2 protein sequences came from the NCBI database. Including: S, E, N, M, ORF3a, ORF8, ORF7a, ORF7b, ORF6, ORF10, orflab, orfla. Among them, the orflab and orfla sequences also included corresponding subsequences. SARS-COV-2 variant's (Delta and Omicro) ORF3 and ORF8 sequences came from 
the NCBI.

2. Related sequences. The related sequence was downloaded from UniProt data set (Table 1).

Table 1. Related sequences are used to search for conserved domains

\begin{tabular}{cllc}
\hline No & Related protein & \multicolumn{1}{c}{ Keywords } & Count \\
\hline 1 & Hemoglobin & Hemoglobin & 56,870 \\
2 & Porphyrin & Bacterial + porphyrin & 30,787 \\
3 & cytochrome c & $\begin{array}{l}\text { Bacterial+ cytochrome + c } \\
\text { Bacterial+heme+oxidase; }\end{array}$ & 111,967 \\
& & ChuS;EfeB;HemS;HmuS;IsdG;MhuD;PhuS & 41325 \\
& HEME Degrade & ShuS;YfeX \\
& & $\begin{array}{l}\text { hemoglobin+protease;Hemoglobin+hydrolysis; } \\
\text { hemoglobin+degrading;hemoglobin+degrade; }\end{array}$ & 2106 \\
5 & Hemoglobin & Hemoglobin+decomposition; & \\
& degrading & Hemoglobin+breakdown & 9780 \\
\hline
\end{tabular}

\subsection{A localized MEME tool to identify conserved domains.}

The following are the steps involved in the analysis:

1. Downloaded MEME from the official website and installed it in a virtual machine running Ubuntu. VM 15 was the virtual machine.

2. Downloaded the SARS-COV-2 protein sequence from the National Center for Biotechnology Information's official website.

3. Obtained the fasta format sequences of the related protein from the official Uniprot website.

4. Generated fasta format files by MEME analysis for each sequence in all related proteins and each SARS-COV-2 protein sequence.

5. To create multiple batches of the files generated in Step 4, a batch size of 50000 was used. It was limitedby the virtual ubuntu system's limited storage space.

6. Using MEME tools in batches, searched for conserved domains $(E-v a l u e<=0.05)$ in SARS-COV-2 and related proteins in Ubuntu.

7. Collected the conserved domains' result files. Located the domain name associated with the motif in the UniProt database.

8. Analyzed the activity of each SARS-COV-2 protein's domains.

\section{RESULTS}

\subsection{Viral proteins possessed the ability to bind to porphyrin and generate heme.}

Porphyrin biosynthesis is the process in mitochondria and cytoplasm. The initial stages of production is in Mitochondria. Three enzymes are involved in the production process: aminolevulinic acid synthase, ALAS1, and ALAS2. In the cytoplasm, the intermediate synthesis stage occurs. Porphobilinogen synthase, porphyrinogen deaminase, uroporphyrinogen III synthetase, and uroporphyrinogen III decarboxylase work sequentially in the synthesis process. The final stage of synthesis occurs in the mitochondria. The production process is carried out 
sequentially by coproporphyrinogen III oxidase, protoporphyrinogen oxidase, and ferrochelatase. The final product of ferrochelatase is heme The majority of mitochondrial synthesis sites are transmembrane areas. If viral proteins are capable of synthesizing porphyrins, these enzymes should be systematically possessed. The viral membrane is analogous to the mitochondrial transmembrane area, while the viral cytoplasm is analogous. As a result, viruses may be able to generate porphyrins via structural proteins. Because non-structural proteins contain both transmembrane and non-transmembrane proteins, they may also produce porphyrin.

We obtained porphyrin-related sequences from the UniProt database and then compared the viral proteins to the porphyrin-related sequences using the local MEME tool. We combined the motif sequences by protein and domain due to the vast number of motif pieces. Table 2 and Table 3 summarizes the search results. Table 2 shows structural proteins possess enzymes synthesized heme. Table 3 shows non-structural proteins possess enzymes synthesized heme. CysG_dimeriser domain has ferrochelatase activity. Elp3 has the function of coproporphyrinogen III oxidase. GlutR_dimer and GlutR_N are involved in the biosynthesis of tetrapyrrole and have glutamyl-tRNA reductase and dimerization domain activities. HEM4 has uroporphyrinogen III synthase activity. HemY_N is a bacterial HemY porphyrin biosynthetic protein, which can oxidize coproporphyrinogen III and protoporphyrinogen IX. Porphobil_deam and Porphobil_deamC are porphobilinogen deaminase. TP_methylase is a tetrapyrrole methylase. Uroporphyrinogen_deCOase has uroporphyrinogen decarboxylase activity.

Table 2. SARS-COV-2 structural proteins possess enzymes synthesized heme

\begin{tabular}{|c|c|c|c|c|}
\hline Protein & Domain & Motif & Start & End \\
\hline \multirow[t]{23}{*}{$\mathrm{S}$} & CysG_dimeriser & KRVDFCGKGYH & 1038 & 1048 \\
\hline & & ELGKYEQYIKWPWYIW & 1202 & 1217 \\
\hline & Elp3 & WFHAIH & 64 & 69 \\
\hline & & YYHKNNKSWMESEFRVYSSANNCTFEYVSQPFLMDL & 144 & 186 \\
\hline & & EGKQGNF & & \\
\hline & & KMSECVLGQSKRVDFCGKGYHLMSFPQSAPH & 1028 & 1058 \\
\hline & & QELGKYEQYIKWPWYIWLGFIAGLIAIVMVTIMLCCM & 1201 & 1269 \\
\hline & & TSCCSCLKGCCSCGSCCKFDEDDSEPVLKGVK & & \\
\hline & GlutR_N & WFHAIH & 64 & 69 \\
\hline & & HWFVTQRNFYEPQ & 1101 & 1113 \\
\hline & & KYEQYIKWPWYIW & 1205 & 1217 \\
\hline & HEM4 & HKNNKSWM & 146 & 153 \\
\hline & & KYEQYIKWPWYIWLGFIAGLIAIVMVTIMLCCMTSCCS & 1205 & 1258 \\
\hline & & CLKGCCSCGSCCKFDE & & \\
\hline & HemY_N & GKYEQYIKWPWYIWLGFIAGLIAIVMVTIMLCCMTSC & 1204 & 1255 \\
\hline & & CSCLKGCCSCGSCCK & & \\
\hline & Porphobil_deam & VYYHKNNKSWM & 143 & 153 \\
\hline & & KYEQYIKWPWYIWLGFIAGLIAIVMVTIMLCCM & 1205 & 1237 \\
\hline & Porphobil_deam & YYHKNNKSWM & 144 & 153 \\
\hline & $\mathrm{C}$ & KYEQYIKWPWYIWLGFIAGLIAIVMVTIMLCCMTSCCS & 1205 & 1254 \\
\hline & & CLKGCCSCGSCC & & \\
\hline & TP_methylase & MQMAYR & 900 & 905 \\
\hline & & GKYEQYIKWPWYIWLGFIAGLIAIVMVTIMLCCMTSC & 1204 & 1248 \\
\hline
\end{tabular}




\begin{tabular}{|c|c|c|c|c|}
\hline & & CSCLKGCC & & \\
\hline & Uroporphyrinog & TWFHAIHV & 63 & 70 \\
\hline & en_deCOase & YYHKNNKSWMESEFR & 144 & 158 \\
\hline & & WNRKRIS & 353 & 359 \\
\hline & & PFAMQMAYR & 897 & 905 \\
\hline & & CGKGYHLM & 1043 & 1050 \\
\hline & & PAICHDGKAHFPREGVFVSNGTHWFVTQRNFYEP & 1079 & 1112 \\
\hline & & IDLQELGKYEQYIKWPWYIWLGFIAGLIAIVMVTIMLC & 1198 & 1260 \\
\hline & & CMTSCCSCLKGCCSCGSCCKFDEDD & & \\
\hline $\mathrm{E}$ & CysG_dimeriser & LCAYCCNIVN & 39 & 48 \\
\hline & Elp3 & TLAILTALRLCAYCCNIVNVSLVKPSFYVYSRVKNLNS & 30 & 68 \\
\hline & & $\mathrm{S}$ & & \\
\hline & GlutR_dimer & CAYCCNIVNVSLVKPSFY & 40 & 57 \\
\hline & GlutR_N & TALRLCAYCCNIVNVSLVKPSFYVYSRVKNLNS & 35 & 67 \\
\hline & HEM4 & RLCAYCCNIVNVSLVKPSFYVYSRVKN & 38 & 64 \\
\hline & HemY_N & CAYCCNIVNVSLVKPSFYVYSRVK & 40 & 63 \\
\hline & Porphobil_deam & ILTALRLCAYCCNIVNVSLVKPSFYVYSRVKNLNSS & 33 & 68 \\
\hline & Porphobil_deam & TALRLCAYCCNIVNVSLVKPSFYVYSRVKNLNSS & 35 & 68 \\
\hline & $\mathrm{C}$ & & & \\
\hline & TP_methylase & RLCAYCCNIVNVSLVKPSFYVYSRVKNLNSSRVPD & 38 & 72 \\
\hline & Uroporphyrinog & TLAILTALRLCAYCCNIVNVSLVKPSFYVYSRVKNLNS & 30 & 69 \\
\hline & en_deCOase & SR & & \\
\hline M & Elp3 & SMWSFNPETNILLNVPLH & 108 & 125 \\
\hline & & HLRIAGHHLGRCDIKDLPKEITVATS & 148 & 173 \\
\hline & & YSRYRIGNYKLNTDHSSSSDN & 196 & 216 \\
\hline & GlutR_N & MACLVGLMW & 84 & 92 \\
\hline & & MWSFNPE & 109 & 115 \\
\hline & HEM4 & MWSFNPET & 109 & 116 \\
\hline & Porphobil_deam & MWSFNPE & 109 & 115 \\
\hline & & HHLGRCDIKDLPKE & 154 & 167 \\
\hline & TP_methylase & CDIKDLPKEITVATSRTLSYYK & 159 & 180 \\
\hline & & KLNTDHSSSSDNI & 205 & 217 \\
\hline & Uroporphyrinog & SMWSFNPE & 108 & 115 \\
\hline & en_deCOase & HHLGRCD & 154 & 160 \\
\hline $\mathrm{N}$ & Elp3 & NNTASWFTALTQH & 47 & 59 \\
\hline & & DLKFPRGQGVPINTNSSPDDQIGYYRRATRRIRGGDGK & 63 & 146 \\
\hline & & MKDLSPRWYFYYLGTGPEAGLPYGANKDGIIWVATE & & \\
\hline & & GALNTPKDHI & & \\
\hline & & IRQGTDYKHWPQIAQFAPSASAFFGMSRIGMEVTPSGT & 292 & 379 \\
\hline & & WLTYTGAIKLDDKDPNFKDQVILLNKHIDAYKTFPPTE & & \\
\hline & & PKKDKKKKADET & & \\
\hline & GlutR_N & MKDLSPRWYFYYLGTGPE & 101 & 118 \\
\hline & & IRQGTDYKHWPQI & 292 & 304 \\
\hline & & SGTWLTYTGAIKLDDKDPNFKDQVILLNKHIDAY & 327 & 360 \\
\hline
\end{tabular}


HEM4

$\begin{array}{lcc}\text { MKDLSPRWYFYYLG } & 101 & 114 \\ \text { DYKHWPQI } & 297 & 304 \\ \text { FKDQVILLNKHIDAYKTF } & 346 & 363 \\ \text { RWYFYYL } & 107 & 113 \\ \text { DYKHWPQIAQFAPSASAFFGMSRIGMEV } & 297 & 324 \\ \text { KHIDAYKTFPP } & 355 & 365 \\ \text { MKDLSPRWYFYYL } & 101 & 113 \\ \text { IRQGTDYKHWPQIAQFAPSASAFFGMSRIGMEV } & 292 & 324 \\ \text { GDGKMKDLSPRWYFYYL } & 97 & 113 \\ \text { PRQKRTATKAYNVTQAFGRRGPEQTQGNFGDQELIRQ } & 258 & 305 \\ \text { GTDYKHWPQIA } & & \\ \text { MSRIGMEVTPSGTWLTYT } & 317 & 334\end{array}$

Porphyrinogen deaminase, uroporphyrinogen III synthase, uroporphyrinogen III decarboxylase, and coproporphyrinogen III oxidase domains are present in both structural and non-structural proteins, as shown in Table 2 and Table 3. There were ferrochelatase domain present. It indicates that structural and non-structural proteins can employ porphobilinogen as a starting material for synthesizing heme, respectively. Multiple viral proteins collaborate to finish the porphyrin production pathway. Due to the limitations of our analysis capabilities, we cannot determine the relation of porphyrin synthesis between viral proteins. These enzyme activity domains are linked to porphyrin. As a result, viral proteins can also interact with porphyrins via these enzyme domains.

Table 3. SARS-COV-2 non-structural proteins possess enzymes synthesized heme

\begin{tabular}{|c|c|c|c|c|}
\hline Protein & Domain & Motif & Start & End \\
\hline \multirow[t]{17}{*}{ ORF3a } & CysG_dimeriser & CWKCRSKNPLLYDANYFLCWHTNCYDYCIPY & 130 & 160 \\
\hline & \multirow[t]{2}{*}{ Elp3 } & QSINFVRIIMRLWLCWKCRSKNPLLYDANYFLCWHTN & \multirow[t]{2}{*}{116} & \multirow[t]{2}{*}{163} \\
\hline & & CYDYCIPYNSV & & \\
\hline & \multirow[t]{2}{*}{ GlutR_N } & IMRLWLCWKCRSKNPLLYDANYFLCWHTNCYDYCIP & \multirow[t]{2}{*}{124} & \multirow[t]{2}{*}{163} \\
\hline & & YNSV & & \\
\hline & \multirow[t]{2}{*}{ HEM4 } & NFVRIIMRLWLCWKCRSKNPLLYDANYFLCWHTNCY & \multirow[t]{2}{*}{119} & \multirow[t]{2}{*}{161} \\
\hline & & DYCIPYN & & \\
\hline & \multirow[t]{2}{*}{ HemY_N } & WKCRSKN & 131 & 137 \\
\hline & & NYFLCWHTNCYDYCIPYN & 144 & 161 \\
\hline & \multirow[t]{2}{*}{ Porphobil_deam } & MRLWLCWKCRSKNPLLYDANYFLCWHTNCYDYCIPY & \multirow[t]{2}{*}{125} & \multirow[t]{2}{*}{162} \\
\hline & & NS & & \\
\hline & \multirow[t]{2}{*}{ Porphobil_deamC } & MRLWLCWKCRSKNPLLYDANYFLCWHTNCYDYCIPY & \multirow[t]{2}{*}{125} & \multirow[t]{2}{*}{161} \\
\hline & & $\mathrm{N}$ & & \\
\hline & \multirow[t]{2}{*}{ TP_methylase } & NFVRIIMRLWLCWKCRSKNPLLYDANYFLCWHTNCY & \multirow[t]{2}{*}{119} & \multirow[t]{2}{*}{161} \\
\hline & & DYCIPYN & & \\
\hline & \multirow{2}{*}{$\begin{array}{l}\text { Uroporphyrinogen } \\
\text { deCOase }\end{array}$} & YFLQSINFVRIIMRLWLCWKCRSKNPLLYDANYFLCW & \multirow[t]{2}{*}{113} & \multirow[t]{2}{*}{162} \\
\hline & & HTNCYDYCIPYNS & & \\
\hline \multirow[t]{4}{*}{ ORF6 } & CysG_dimeriser & WNLDYIIN & 27 & 34 \\
\hline & \multirow[t]{2}{*}{ Elp3 } & MFHLVDFQVTIAEILLIIMRTFKVSIWNLDYIINLIIKNLS & \multirow[t]{2}{*}{1} & \multirow[t]{2}{*}{61} \\
\hline & & KSLTENKYSQLDEEQPMEID & & \\
\hline & GlutR_N & MFHLVDFQVTIAEILLIIMRTFKVSIWNLDYIINLIIKNLS & 1 & 61 \\
\hline
\end{tabular}




\begin{tabular}{|c|c|c|c|c|}
\hline & & KSLTENKYSQLDEEQPMEID & & \\
\hline & HEM4 & MFHLVDFQVTIAEILLIIMRTFKVSIWNLDYIINLIIKNLS & 1 & 58 \\
\hline & & KSLTENKYSQLDEEQPM & & \\
\hline & HemY_N & FQVTIAEILLIIMRTFKVSIWNLDYIINLIIKNLSKSLTEN & 7 & 50 \\
\hline & & KYS & & \\
\hline & Porphobil_deam & MFHLVDFQVTIAEILLIIMRTFKVSIWNLDYIINLIIKNLS & 1 & 59 \\
\hline & & KSLTENKYSQLDEEQPME & & \\
\hline & Porphobil_deamC & TFKVSIWNLDYIINLIIKNLSKSLTENKYSQLDEEQPME & 21 & 59 \\
\hline & TP_methylase & MFHLVDFQVTIAEILLIIMRTFKVSIWNLDYIINLIIKNLS & 1 & 61 \\
\hline & & KSLTENKYSQLDEEQPMEID & & \\
\hline & Uroporphyrinogen & MRTFKVSIWNLDYIINLIIKNLSKSLTENKYSQLDEEQP & 19 & 59 \\
\hline & _deCOase & $\mathrm{ME}$ & & \\
\hline ORF7a & Elp3 & TCELYHYQECVRGT & 14 & 27 \\
\hline & & TYEGNSPFHPLADNKFALTCFSTQFAFACPDGVKHVY & 39 & 76 \\
\hline & & Q & & \\
\hline & GlutR_N & TCELYHYQECVRGTTVLLKEPCSSGTYEGNSPFHPLAD & 14 & 87 \\
\hline & & NKFALTCFSTQFAFACPDGVKHVYQLRARSVSPKLF & & \\
\hline & HEM4 & TCELYHYQEC & 14 & 23 \\
\hline & & KEPCSSGTYEGNSPFHPLADNKF & 32 & 54 \\
\hline & & QFAFACPDGVKHVYQ & 62 & 76 \\
\hline & HemY_N & PCSSGTYEGNSPFHPLADNKFALTCFSTQFAFAC & 34 & 67 \\
\hline & Porphobil_deam & CELYHYQECVRGTTVLLKEPCS & 15 & 36 \\
\hline & Porphobil_deamC & YHYQEC & 18 & 23 \\
\hline & & NSPFHPLADNKFALTC & 43 & 58 \\
\hline & TP_methylase & YHYQEC & 18 & 23 \\
\hline & & YEGNSPFHPLADNKFALTCFSTQ & 40 & 62 \\
\hline & & SVSPKLFIRQEEVQELYSPI & 81 & 100 \\
\hline & Uroporphyrinogen & ATCELYHYQECVRGTTVLLKEPCSSGTYEGNSPFHPLA & 13 & 78 \\
\hline & _deCOase & DNKFALTCFSTQFAFACPDGVKHVYQLR & & \\
\hline ORF7b & CysG_dimeriser & DFYLCFLAFLLFLVLIMLIIFWFSLELQDHNETCH & 8 & 42 \\
\hline & Elp3 & MIELSLIDFYLCFLAFLLFLVLIMLIIFWFSLELQDHNET & 1 & 42 \\
\hline & & $\mathrm{CH}$ & & \\
\hline & GlutR_N & MIELSLIDFYLCFLAFLLFLVLIMLIIFWFSLELQDHNET & 1 & 42 \\
\hline & & $\mathrm{CH}$ & & \\
\hline & HEM4 & MIELSLIDFYLCFLAFLLFLVLIMLIIFWFSLELQDHNET & 1 & 43 \\
\hline & & $\mathrm{CHA}$ & & \\
\hline & HemY_N & IDFYLCFLAFLLFLVLIMLIIFWFSLELQDHNETCH & 7 & 42 \\
\hline & Porphobil_deam & MIELSLIDFYLCFLAFLLFLVLIMLIIFWFSLELQDHNET & 1 & 43 \\
\hline & & CHA & & \\
\hline & Porphobil_deamC & IMLIIFWFSLELQDHNETCHA & 23 & 43 \\
\hline & TP_methylase & MIELSLIDFYLCFLAFLLFLVLIMLIIFWFSLELQDHNET & 1 & 43 \\
\hline & & CHA & & \\
\hline & Uroporphyrinogen & MIELSLIDFYLCFLAFLLF & 1 & 19 \\
\hline
\end{tabular}




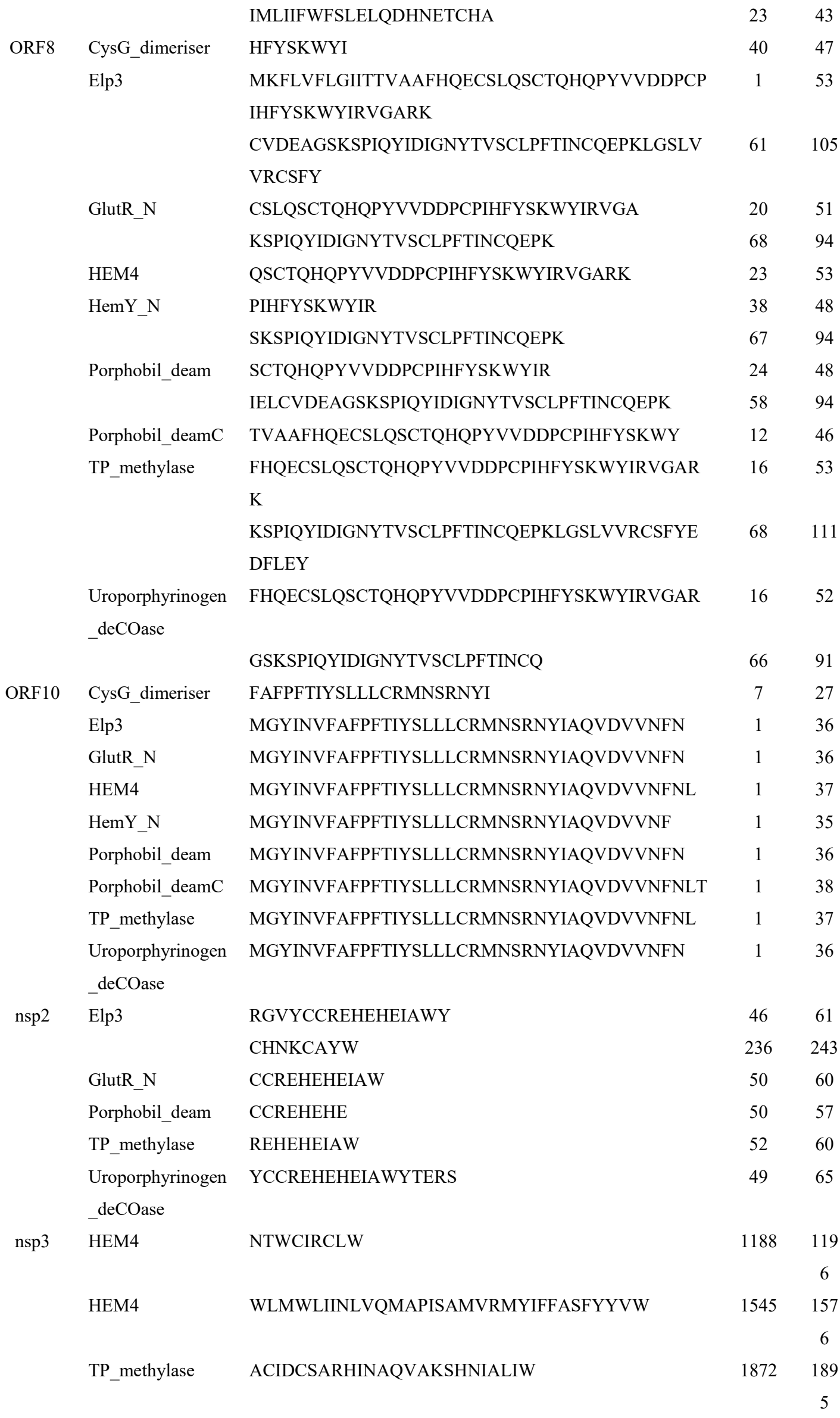




\begin{tabular}{|c|c|c|c|c|}
\hline & $\begin{array}{l}\text { Uroporphyrinogen } \\
\text { _deCOase }\end{array}$ & GRYMSALNHTKKWKYPQVNGLTSIKWADNNCY & 826 & 857 \\
\hline \multirow[t]{2}{*}{ nsp4 } & Elp3 & AHIQWMVMFTPLVPFWITIAYIICISTKHFYWFFS & 361 & 395 \\
\hline & HEM4 & AHIQWMVMFTPLVPFWITI & 361 & 379 \\
\hline \multirow[t]{2}{*}{ nsp6 } & CysG_dimeriser & WVMRIMTW & 90 & 97 \\
\hline & $\begin{array}{l}\text { Uroporphyrinogen } \\
\text { deCOase }\end{array}$ & AYFNMVYMPASWVMRIMTWLDM & 79 & 100 \\
\hline \multirow[t]{2}{*}{ nsp10 } & Elp3 & SCCLYCRCHIDHPN & 72 & 85 \\
\hline & HEM4 & YCRCHIDHPNP & 76 & 86 \\
\hline 2'-O-rib & Elp3 & AMPNLYKMQRM & 10 & 20 \\
\hline $\begin{array}{c}\text { ose } \\
\text { methyltr } \\
\text { ansferas } \\
\text { e }\end{array}$ & $\begin{array}{l}\text { Uroporphyrinogen } \\
\text { _deCOase }\end{array}$ & MGHFAWW & 184 & 190 \\
\hline $\begin{array}{l}\text { 3C-like } \\
\text { proteina } \\
\text { se }\end{array}$ & GlutR_N & YMHHME & 161 & 166 \\
\hline 3'-to-5' & CysG_dimeriser & AKPPPGDQFKHLIPLMYKGLPW & 138 & 159 \\
\hline \multirow[t]{9}{*}{$\begin{array}{l}\text { exonucl } \\
\text { ease }\end{array}$} & Elp3 & $\begin{array}{l}\text { CWHHSIGFDYVYNPFMIDVQQWGFTGNLQSNHDLYC } \\
\text { QVHGNAH }\end{array}$ & 226 & 268 \\
\hline & GlutR_N & YACWHH & 224 & 229 \\
\hline & HEM4 & GYPNMFITREEAIRHVRAW & 68 & 86 \\
\hline & & WHHSIGFDYVYNPFMIDVQQWGFTGNLQSNH & 227 & 257 \\
\hline & & HHANEYRLYLDAYNMMISAGFSLWVYKQF & 486 & 514 \\
\hline & TP_methylase & PPPGDQFKHLIPLMYKGLPWNVVR & 140 & 163 \\
\hline & $\begin{array}{l}\text { Uroporphyrinogen } \\
\text { deCOase }\end{array}$ & $\begin{array}{l}\text { MGFKMNYQVNGYPNMFITREEAIRHVRAWIGFDVEG } \\
\mathrm{CH}\end{array}$ & 58 & 95 \\
\hline & & WHHSIG & 227 & 232 \\
\hline & & CRHHANEY & 484 & 491 \\
\hline \multirow[t]{2}{*}{ helicase } & Elp3 & RCGACIRRPFLCCKCCYDHVISTSHK & 15 & 40 \\
\hline & $\begin{array}{l}\text { Uroporphyrinogen } \\
\text { deCOase }\end{array}$ & GPDMFLGTCRRC & 433 & 444 \\
\hline $\begin{array}{l}\text { leader } \\
\text { protein }\end{array}$ & $\begin{array}{l}\text { Uroporphyrinogen } \\
\text { deCOase }\end{array}$ & PHGHVM & 80 & 85 \\
\hline \multirow[t]{3}{*}{ RdRP } & Elp3 & PHEFCSQHTMLVKQGDDYVYLPYP & 809 & 832 \\
\hline & Porphobil_deam & ENPHLMGWDYPKCDRAMPNM & 610 & 629 \\
\hline & $\begin{array}{l}\text { Uroporphyrinogen } \\
\text { deCOase }\end{array}$ & YSDVENPHLMGWDYPKCDRAMPNMLRIMA & 606 & 634 \\
\hline
\end{tabular}

\subsection{The Haem_bd domain of viral proteins could bind heme.}

The UniProt database was used to find cytochrome C-related sequences. Then we adopted the local version of MEME to match the viral proteins and cytochrome C-related sequences. Due to a large number of motif fragments, we organized motif sequences by protein and domain. Table 4 displays the search results. S, N, E, ORF3a, ORF7b, ORF10, nsp2, nsp3, and RdRP all have Haem_bd domains, as seen in Table 4. A possible heme-binding motif $\mathrm{CXXCH}$ can be found in 
the Haem_bd (PF14376) domain. H connects to the iron in heme, while two Cs bind to porphyrin(92). The Haem_bd domain is found in most cytochrome $\mathrm{C}$ oxidases. It demonstrates that the Haem bd domain of these viral proteins can bind heme.

Table 4. Haem bd domain of SARS-COV-2 viral protein

\begin{tabular}{cclcc}
\hline Protein & Alias & \multicolumn{1}{c}{ Haem_bd Motif } & Start & END \\
\hline S & A & WFHAIH & 64 & 69 \\
& B & NFTTAPAICHDGKAHFPRE & 1074 & 1092 \\
& C & GKYEQYIKWPWYIWLGFIAGLIAIVMVTIMLCCM & 1204 & 1237 \\
E & A & RLCAYCC & 38 & 44 \\
N & A & PRWYFYYLGTGPEAGLPYGANKDGIIWVATEGALNTPKDH & 106 & 145 \\
& B & QGTDYKHWPQIAQFAPSASAFFGMSRIGMEVTPSGTWLTY & 294 & 333 \\
& C & VILLNKHIDAYKTFPPTE & 350 & 367 \\
orf3a & A & NFVRIIMRLWLCWKCRS & 119 & 135 \\
& B & NCYDYC & 152 & 157 \\
ORF7b & A & QDHNETCH & 35 & 42 \\
ORF10 & A & CRMNSRNY & 19 & 26 \\
nsp2 & A & KRGVYCCREHEHEIAWYTERSEKSYELQTPFE & 45 & 76 \\
nsp3 & A & CASEYTGNYQCGHYKHI & 1005 & 1021 \\
RdRP & A & PHLMGWDYPKCDRAMPNMLRIMASLVLARKH & 612 & 642 \\
\hline
\end{tabular}

According to the motif $\mathrm{CXXCH}$ of the linked sequences, we formed a one-to-one correlation with the results in Table 4, and then sorted out the Haem motif (Table 5). Only E and ORF3a carry the CXXC motif, as shown in Table 5. Hematoporphyrin binds both $\mathrm{C}$ molecules. E has $\mathrm{C} 44$ as $\mathrm{Fe}$ binding sites, and ORF3a has R134. But neither does $\mathrm{H}$. This mutation may make it easier for viral proteins to bind iron. E and ORF3a bind to heme in a relatively steady manner. Other viral proteins' heme-binding could be unstable.

Table 5. The Haem motif of CXXCH is in the SARS-COV-2 viral protein.

\begin{tabular}{ccccc}
\hline Protein & Alias & Haem motif & Start-End & Haem-Fe Site \\
\hline S & A & PAICH & $1079-1083$ & H1083 \\
& B & KYEQY & $1205-1209$ & Y1209 \\
E & A & CAYCC & $40-44$ & C44 \\
N & A & WYFYY, YYLGT & $108-112,111-115$ & Y112, T115 \\
& B & TDYKH, DYKHW & $296-300,297-301$ & H300, W301 \\
& C & NKHID, HIDAY & $354-358,356-360$ & D358, Y360 \\
ORF3a & A & CWKCR & $130-134$ & R134 \\
& B & CYDYC & $153-157$ & C157 \\
ORF7b & A & NETCH & $38-42$ & H42 \\
nsp2 & A & VYCCR & $228-232$ & R232 \\
nsp3 & A & CASEY & $1823-1827$ & Y1827 \\
\hline
\end{tabular}

\subsection{Viral proteins could bind hemoglobin}

Eryth_link_C is on the linker subunit of the giant extracellular hemoglobin (globin) respiratory complex. The linker subunit's C-terminal globular domain is involved in trimerization. It also interacts with globin and other adjacent trimers' C-terminal spherical linker domains. In Staphylococcus aureus, the NEAT domain encodes the human hemoglobin receptor. The NEAT 
domain recognizes a subfamily of iron-regulated surface determinant proteins that are found exclusively in bacteria. Iron-regulated surface determining protein $\mathrm{H}$ (isdH, also known as harA) interacts with the human plasma haptoglobin-hemoglobin complex, haptoglobin, and hemoglobin. It has a much higher affinity for haptoglobin-hemoglobin complexes than haptoglobin alone. These three domains serve distinct purposes. IsdH(N1) binds hemoglobin and haptoglobin; IsdH(N3) binds heme that has been released from hemoglobin.

Table 6. The SARS-COV-2 virus protein contains the Eryth_link_C and NEAT domains.

\begin{tabular}{|c|c|c|c|c|c|}
\hline Protein & Domain & Alia & Motif & Start & End \\
\hline \multirow[t]{3}{*}{$\mathrm{S}$} & Eryth_link_C & A & CEFQFCNDPFLGVYYHKNNKSWMESEFR & 131 & 158 \\
\hline & & $\mathrm{B}$ & YIKWPWYIWL & 1209 & 1218 \\
\hline & NEAT & A & KWPWYIWLGFIAGLIAIVMVTIMLCCMT & 1211 & 1238 \\
\hline $\mathrm{E}$ & NEAT & A & LRLCAYCC & 37 & 44 \\
\hline \multirow[t]{3}{*}{$\mathrm{N}$} & Eryth_link_C & A & YKHWPQIAQF & 298 & 307 \\
\hline & NEAT & A & RWYFYY & 107 & 112 \\
\hline & & $\mathrm{B}$ & ELIRQGTDYKHWPQI & 290 & 304 \\
\hline \multirow[t]{2}{*}{ ORF3a } & NEAT & A & NFVRIIMRLWLCWKCRSKNPLLYDANYFLC & 119 & 158 \\
\hline & & & WHTNCYDYCI & & \\
\hline \multirow[t]{3}{*}{ ORF6 } & Eryth_link_C & $\mathrm{A}$ & ILLIIMRTFKVSIWNLDYIINLIIKNLSKSLTEN & 14 & 60 \\
\hline & & & KYSQLDEEQPMEI & & \\
\hline & NEAT & A & ILLIIMRTFKVSIWNLDYIINLIIKN & 14 & 39 \\
\hline ORF7a & NEAT & A & CELYHYQECVR & 15 & 25 \\
\hline \multirow[t]{2}{*}{ ORF7b } & Eryth_link_C & A & MLIIFWFSLELQDHNETCH & 24 & 42 \\
\hline & NEAT & A & MLIIFWFSLELQDHNETCHA & 24 & 43 \\
\hline \multirow[t]{4}{*}{ ORF8 } & Eryth_link_C & A & AFHQECSLQSCTQHQPYVVDDPCPIHFYSKW & 15 & 53 \\
\hline & & & YIRVGARK & & \\
\hline & NEAT & $\mathrm{A}$ & FHQECSLQSCTQHQPYVVDDPCPIHFYSKWYI & 16 & 75 \\
\hline & & & RVGARKSAPLIELCVDEAGSKSPIQYID & & \\
\hline \multirow[t]{4}{*}{ ORF10 } & Eryth_link_C & A & MGYINVFAFPFTIYSLLLCRMNSRNYIAQVDV & 1 & 35 \\
\hline & & & VNF & & \\
\hline & NEAT & A & MGYINVFAFPFTIYSLLLCRMNSRNYIAQVDV & 1 & 36 \\
\hline & & & VNFN & & \\
\hline nsp6 & Eryth_link_C & $\mathrm{A}$ & FNMVYMPASWVMRIMTW & 81 & 97 \\
\hline nsp10 & Eryth_link_C & A & FGGASCCLYCRCHIDH & 68 & 83 \\
\hline RdRP & Eryth_link_C & A & ENPHLMGWDYPKCDRAMPNMLRIM & 610 & 633 \\
\hline 2'-O-ribose & NEAT & $\mathrm{A}$ & MGHFAWWTAF & 184 & 193 \\
\hline \multicolumn{6}{|l|}{ methyltrans } \\
\hline ferase & & & & & \\
\hline
\end{tabular}

We downloaded hemoglobin-related sequences from the UniProt database. We then compared the viral proteins to the hemoglobin-related sequences using the local MEME version. We combined the motif sequence by protein and domain due to the vast number of motif pieces. Table 6 summarizes the search results. S, E, N, ORF3a, ORF6, ORF7a, ORF7b, ORF8, ORF10, 2'-O-ribose methyltransferase contain NEAT domains, as shown in Table 6. The domain Eryth_link_C is present in S, N, ORF6, ORF7b, ORF8, ORF10, nsp6, nsp10, and RdRP. Both Eryth link $\mathrm{C}$ and NEAT domains are in S, N, ORF6, ORF7b, ORF8, ORF10, respectively. Because 
$\mathrm{S}$ can form a trimer structure, it can be combined with extracellular hemoglobin. The $\mathrm{S}$ Eryth_link_C A is involved in receptor binding. S Eryth_link_C B is a member of the S2 family of proteins involved in membrane integration. The NEAT domains of S, E, N, ORF3a, ORF6, ORF7a, ORF7b, ORF8, ORF10, and 2'-O-ribose methyltransferase may perform distinct functions. S NEAT A and S Eryth_link_C B are mutually overlapped. E NEAT A is shorter proteins that perform the function of heme capture. S, N, ORF3a, ORF6, ORF7b, ORF8, ORF10 have a longer NEAT capable of binding and capturing hemoglobin.

\subsection{ORF3a acted as a hemoglobin protease.}

The autotransporter subfamily has two peptidase S6 domains (IPR030396 and PS51691). S06.001 - Neisseria type serine peptidase specific for IgA1, MEROPS accession number MER0000278. Peptidase S6 domains are found in the IgA-specific serine endopeptidase autotransporter of Neisseria gonorrhoeae, the immunoglobulin A1 protease autotransporter of Haemophilus influenzae, and the hemoglobin binding protease Hbp autotransporter of pathogenic Escherichia coli. In contrast to IgA peptidases (Family S6 contains Neisseria and Haemophilus IgA1-specific endopeptidases), Enterobacter sp. (SPATE) peptidases have not been demonstrated to cleave IgA1. SPATE proteins are all highly immunogenic, and each SPATE member is one of the pathogen's most prominent secreted proteins. Human $\operatorname{IgA} 1$ is cleaved by the bacterial $\operatorname{IgA} 1$ protease at a hinge region where IgA2 is not present. They are highly selective prolyl endopeptidases, and the site of cleavage within human IgAl's hinge region differs between strains. Hbp attaches to hemoglobin, destroys it, and then binds to the heme that is liberated.

His, Asp, Ser, in that order, are the catalytic residues of the S6 family. Ser is found in the Gly-Xaa-Ser-Gly-Xaa-Pro motif, which is highly conserved across the S1-S2 families and the S7, S29, and S30 families. S6 is a member of the PA clan as a result of this. As established experimentally in the Hap protein of Haemophilus influenza, his and asp residues are positioned around 190 and 120 residues N-terminal to the catalytic Ser. By cleaving human IgA1, The protective role of the powerful medium, IgA1 serine peptidase, may disrupt mucosal surface-specific immunity.

We used a local version of MEM to compare the hemoglobin degrading-related sequences to the SARS-COV-2 viral protein to identify conserved structures. We combined the motif fragments according to viral protein and domain classification. Table 7 illustrates the domain distribution of Peptidase S6 in SARs-COV-2. Peptidase S6 domains are shown in Table 7 for E, ORF3a, ORF7a, ORF7b, ORF8, and ORF10. However, only the ORF3a Peptidase S6 domains share a similar Gly-Xaa-Ser-Gly-Xaa-Pro motif: C-R-S-K-N-P. C and K are unambiguously derived from Gly or G. This mutation could be used to improve ORF3a's affinity for heme. As determined by heme motif research, $\mathrm{CR}$ is the final two letters $\mathrm{CH}$ in the Haem bind domain's $\mathrm{CXXCH}$ motif. $\mathrm{C}$ denotes heme's porphyrin-binding site, while $\mathrm{R}$ denotes iron-binding. It indicates that ORF3a acts on hemoglobin via the Peptidase S6 domains of the hemoglobin protease. The catalytic site C-R-S-K-N-P acts on the heme on hemoglobin, and then hunts for and binds to heme directly. ORF3a, ORF8, S, and N, as well as others, have a more significant number of autotransporter domains(90). As a result, ORF3a functioned as an IgA1 peptidase and a hemoglobin protease. 
Table 7. The SARS-COV-2 virus protein contains the Peptidase S6 domain

\begin{tabular}{clccc}
\hline Domain & \multicolumn{1}{c}{ Motif } & Start & End & X-X-Ser-X-X-Pro \\
\hline E & CAYCCNIVNVSLVKP & 40 & 54 & - \\
ORF3a & RIIMRLWLCWKCRSKNPLLYDANYFLCWHTNCYDYCIP & 122 & 159 & CRSKNP \\
ORF7a & HPLADNKFALTCFSTQFAFACPDGVKHVY & 47 & 75 & - \\
ORF7b & MIELSLIDFYLCFLAFLLFLVLIMLIIFWFSLELQDHNETCH & 1 & 42 & - \\
ORF8 & PYVVDDPCPIHFYSKWYIRVGARKSAPLI & 30 & 58 & - \\
ORF10 & MGYINVFAFPFTIYSLLLCRMNSRNY & 1 & 26 & - \\
\hline
\end{tabular}

\subsection{ORF3a protein possessed IsdA, IsdC, and IsdH activity for uptaking heme.}

ORF3a can bind to and degrade hemoglobin, as seen in Tables 6-7. ORF3a can also bind heme, as demonstrated in Tables 4-5. It indicates that ORF3a can bind to and degrade hemoglobin before collecting heme. As seen in Table 6, ORF3a has a NEAT domain. All Isd proteins involved in heme absorption contain NEAT domains. IsdA, IsdC, and IsdH/IsdB proteins are present in Staphylococcus aureus. They are capable of completing activities such as hemoglobin localization and heme capture. Table 8 summarizes the protein sequences corresponding to the ORF3a NEAT domain found in the search results. As shown in Table 8, ORF3a's NEAT domains are derived from IsdA, IsdC, and IsdH. The three proteins' motifs, notably the heme-binding motif "CWKCR," are remarkably similar, indicating that their functions are intimately tied to heme capture. The searches mentioned above show that the IsdH domain supports ORF3a in localizing hemoglobin, and then the IsdA domain attacks hemoglobin. The IsdA domain is responsible for transferring the heme from hemoglobin to IsdC. IsdC binds to heme.

Table 8. ORF3a's NEAT domain come from Isd proteins

\begin{tabular}{llcc}
\hline \multicolumn{1}{c}{ Protein } & \multicolumn{1}{c}{ Motif } & Start & End \\
\hline Cell surface protein IsdA, transfers & NFVRIIMRLWLCWKCRSKNPLLYDANYFLCW & 119 & 157 \\
heme from hemoglobin to apo-IsdC & HTNCYDYC & & 158 \\
Heme uptake protein IsdC & MRLWLCWKCRSKNPLLYDANYFLCWHTNCY & 125 & 158 \\
Iron-regulated surface determinant & DYCI & FVRIIMRLWLCWKCR & 120 \\
protein H ( IsdH) & & & 134 \\
\hline
\end{tabular}

\subsection{ORF3a protein was capable of converting heme to iron and porphyrin.}

The ABM domain (IPR007138) is a monooxygenase domain involved in the biosynthesis of antibiotics. This domain is found in the IsdG and IsdI strains of S. aureus. IsdG and IsdI are heme-degrading enzymes that resemble monooxygenases structurally. This domain is also found in the MhuD heme-degrading monooxygenase from Mycobacterium tuberculosis. HemS/ChuX (IPR007845) is a protein that degrades heme. ABM monooxygenases (IsdG and IsdI) released iron from heme by cleaving the heme tetrapyrrole ring structure in the presence of NADPH cytochrome P450 reductase. HemS catalyzes the release of iron from heme without causing damage to the tetrapyrrole backbone. After HemS binds to heme, the tetrapyrrole ring structure of heme also is broken to release iron in the presence of electron donors such as ascorbate or NADPH-cytochrome P450 reductase.

We retrieved the sequences of proteins involved in heme degradation from the UniProt 
database. Then we compared them one by one to ORF3a using the local MEME version. We combined the discovered motif fragments according to conserved domains. The heme degradation domains of the ORF3a protein searched are listed in Table 9. ORF3a contains ABM and HemS domains, as shown in Table 9. ORF3a ABM and ORF3a HemS were homologous. The N-terminus of ORF3a ABM includes more "NFVRI" sequence fragments than the N-terminus of ORF3a HemS. The C-terminal region of ORF3a HemS has more "YDYCIPYNSV" sequence fragments than the C-terminal region of ORF3a ABM. ORF3a ABM and ORF3a HemS both include the heme-binding motif "CWKCR," indicating that ORF3a ABM and ORF3a HemS can bind heme. As shown in Table 9, the ORF3a protein could directly separate heme into iron and porphyrin via the HemS domain. ORF3a protein could also cleave the tetrapyrrole ring of heme via the $\mathrm{ABM} / \mathrm{HemS}$ domain, thereby degrading heme and releasing iron in the presence of NADPH-cytochrome P450 reductase.

Table 9. ORF3a protein has a heme-degrading domain.

\begin{tabular}{clcc}
\hline Domain & \multicolumn{1}{c}{ Motif } & Start & End \\
\hline ABM & NFVRIIMRLWLCWKCRSKNPLLYDANYFLCWHTNC & 119 & 153 \\
HemS & IMRLWLCWKCRSKNPLLYDANYFLCWHTNCYDYCIPYNSV & 124 & 163 \\
\hline
\end{tabular}

\subsection{ORF3a protein impaired human respiratory function}

We retrieved the crystal structure of ORF3a from the PDB database and then annotated the conserved domains that attack hemoglobin and degrade heme using the Discovery Studio 2016 tool (Figure 1). ORF3a is a dimer in Figure 1. The heme-binding motifs "CWKCR" and "CYDYC" of a monomeric ORF3a are labeled in Figure 1.A. Coincidentally, the two phantoms are positioned adjacent, producing a clip. It indicated that the clip's function was to capture the heme and expel the iron. The NEAT domain of a monomeric ORF3a is labeled in Figure 1.B. The NEAT structure comprises densely packed IsdA, IsdC, and IsdH domains. The NEAT domain contains the heme-binding motif "CWKCR" and "CYDYC". The Peptidase S6 domain of a monomeric ORF3a is labeled in Figure 1.C. The Peptidase S6 domain spans the heme-binding motif "CWKCR" and "CYDYC". These two heme motifs contain the unusual X-X-Ser-X-X-Pro pattern CRSKNP. The Peptidase S6 domain overlaps the NEAT domain. The HemS domain of a monomeric ORF3a is labeled in Figure 1.D. The HemS domain spans the heme-binding motif "CWKCR" and "CYDYC". The HemS domain is overlaps to the NEAT, Peptidase S6, and ABM domains. It implies that the sequence fragments around the heme-binding motifs "CWKCR" and "CYDYC" are highly conserved. They are involved in hemoglobin assault and heme degradation.

ORF3a may have a mechanism for impairing respiratory function. ORF3a infected erythrocytes via domains like that of Plasmodium falciparum. ORF3a formed ion channels by binding cytoskeletal proteins such as spectrin in red blood cell membranes. The HemS, NEAT, Peptidase S6, and ABM domains were positioned near the cytoplasm in the erythrocyte inner membrane. ORF3a interacted with the hemoglobin protein via the NEAT structure. The NEAT domain possessed the IsdA, IsdC, and IsdH functions. ORF3a might be linked to 1-alpha-Hb via the IsdH domain of the NEAT domain, then the IsdH domain of the NEAT domain was positioned near the heme-binding region of 1-beta-Hb. ORF3a altered the shape of beta-Hb via the Peptidase S6 domain. As a result, heme was lost from 1-beta-Hb. ORF3a IsdA was responsible for capturing shed heme and transporting it to the region of ORF3a IsdC. Then, via the HemS domain, ORF3a dissociated heme into iron and porphyrin. ORF3a cleaved the tetrapyrrole ring of heme via the 
HemS or ABM domain in the presence of NADPH-cytochrome P450 reductase to liberate iron. Due to the Isd domains of ORF3a, hemoglobin attacked by ORF3a might preserve the majority of its natural domain while exhibiting reduced oxygen transport function.
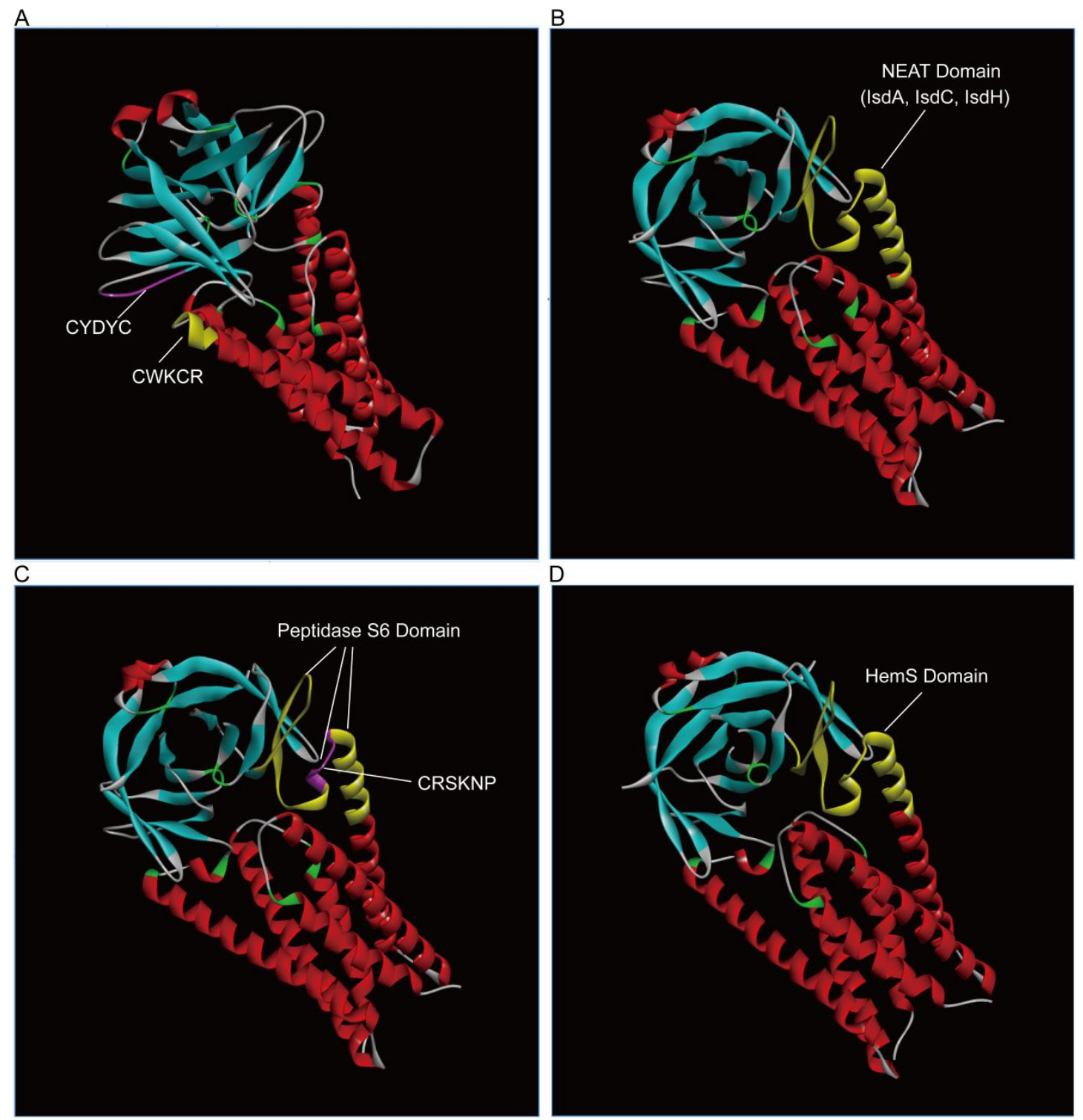

Figure 1. Domains' three-dimensional distribution of ORF3a (PDBID: 6xdc) binding to hemoglobin and cleavage heme. A. ORF3a contains two heme-binding motifs. B. The ORF3a NEAT domain binds hemoglobin, including IsdA, IsdC, and IsdH. C. ORF3a Peptidase S6 domain catalyzes the degradation of the hemoglobin. D. ORF3a HemS domain catalyzes the dissociation of heme into the iron and porphyrin. HemS is overlapped to the ABM domain. In the presence of NADPH-cytochrome P450 reductase, HemS and ABM split the tetrapyrrole ring of heme and liberate iron.

\section{8 $\mathrm{S}$ protein efficiently transported oxygen molecules through hemocyanin activity}

Hemocyanin is commonly found in hexamers and has three copper-binding tyrosinase sites. Because SARs-COV-2 S protein could form hexamers during membrane fusion, we examined whether S protein possesses hemocyanin activity. We obtained the hemocyanin-related sequences 
from the Uniprot database and used the local MEME tool to compare them to the S protein individually. Finally, we will combine the searched motifs according to conserved domains. S hemocyanin-related domains are listed in Table 10.

Table 10. Hemocyanin activity-related domains of SARs-COV-2 S protein

\begin{tabular}{lclcc}
\hline \multicolumn{1}{c}{ Domain } & Alias & \multicolumn{1}{c}{ Motif } & Start & End \\
\hline Hemocyanin_C & A & EFQFCNDPFLGVYYHKNNKSWME & 132 & 154 \\
& B & RVDFCGKGYHLMS & 1039 & 1051 \\
& C & ICHDGKAH & 1081 & 1088 \\
& D & QELGKYEQYIKWPWYIWLGFIAGLIAIVMVTIMLCCM & 1201 & 1256 \\
& & TSCCSCLKGCCSCGSCCKF & & 64 \\
Hemocyanin_M & A & WFHAIH & 144 & 160 \\
& B & YYHKNNKSWMESEFRVY & 738 & 749 \\
& C & CTMYICGDSTEC & 898 & 906 \\
& D & FAMQMAYRF & 1069 & 1093 \\
& E & PAQEKNFTTAPAICHDGKAHFPREG & 1202 & 1244 \\
& F & ELGKYEQYIKWPWYIWLGFIAGLIAIVMVTIMLCCMT & 1211 & 1241 \\
& & SCCSCL & 1079 & 1093 \\
Hemocyanin_N & A & KWPWYIWLGFIAGLIAIVMVTIMLCCMTSCC & 1206 & 1217 \\
& A & PAICHDGKAHFPREG & 1229 & 1253 \\
\hline & B & YEQYIKWPWYIW & C & MVTIMLCCMTSCCSCLKGCCSCGSC
\end{tabular}

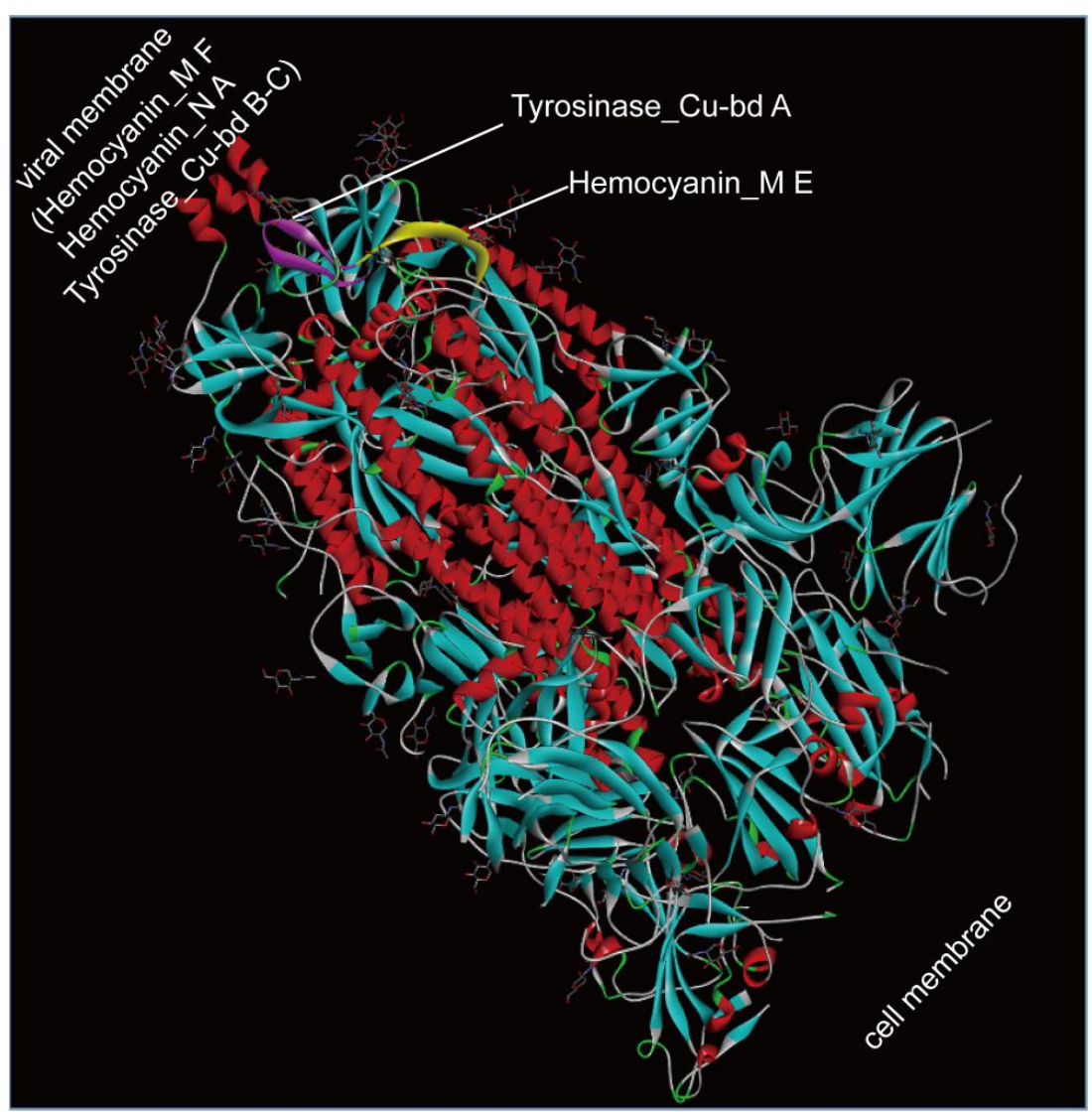

Figure 2. Schematic diagram of the crystal structure positions of the Hemocyanin_M, Hemocyanin_N, and Tyrosinase_Cu-bd domains of the S protein (PDBID: 6vyb). 
According to Table 10, the $\mathrm{S}$ protein contains the Hemocyanin protein's C-terminal (Hemocyanin_C), active (Hemocyanin_M), and N-terminal (Hemocyanin_N) domains. The S protein contains three copper-binding tyrosinase regions (Tyrosinase_Cu-bd A-C). Tyrosinase_Cu-bd A-C are found in the active area of the Hemocyanin protein, Hemocyanin_M domains. Tyrosinase_Cu-bd A resides in Hemocyanin_M E. In contrast, Tyrosinase_Cu-bd B-C resides in Hemocyanin_M F. Hemocyanin_C and Hemocyanin_M are found in the S protein's $\mathrm{N}$ to $\mathrm{C}$ terminus. Hemocyanin_N is only found at the $\mathrm{S}$ protein's C-terminus.

The schematic diagram in Figure 2 illustrates the three-dimensional placements of the domains of Hemocyanin_M, Hemocyanin_N, and Tyrosinase_Cu-bd. Hemocyanin_N A possesses phenoloxidase activity, but it is located in the transmembrane area. It is consistent with phenol oxidase being isolated from phenolic substrates. Hemocyanin_N also serves as the binding site for the enzyme Tyrosinase_Cu-bd B-C. Tyrosinase_Cu-bd A is located on the exterior of the viral membrane. When Tyrosinase_Cu-bd A binds to $\mathrm{Cu}$, it may initiate a chain reaction, causing a conformational shift in $\mathrm{S}$ to expose the Hemocyanin_N domain ThenTyrosinase Cu-bd B-C bind to copper. After Tyrosinase $\mathrm{Cu}$-bd A-C bind copper, $\mathrm{S}$ protein exhibits hemocyanin activity.

\subsection{Functional consequences of the Delta and Omicron mutations on the ORF3a and $S$ proteins}

Delta (B1.617.2) and Omicron (B.1.1.529) mutants are SARs-COV-2 viruses that spread globally starting in 2021. All relevant variants had significantly higher viral loads than the wild type, with Omicron's average viral loads being many times that of Delta(91). Individuals infected with Omicron exhibit only subtle symptoms. Omicron mutants have a more significant number of mutation sites and a greater capacity for immune evasion. We obtained the ORF3a and S proteins from the NCBI database for the Delta and Omicron mutations. Then, the effect of mutations on the ORF3a and S proteins' attack functions was compared.

Mutations in the $\mathbf{S}$ protein alleviated symptoms of respiratory distress in patients. We used the local MEME tool to match the $\mathrm{S}$ proteins of the Delta and Omicron mutants to hemocyanin-related sequences. The discovered motifs were integrated according to domains, and the resulting search results are displayed in Table 11. The S proteins of Delta and Omicron mutants contain Hemocyanin_C, Hemocyanin_M, Hemocyanin_N, and Tyrosinase_Cu-bd domains, as shown in Table 11. The hemocyanin activity-related domains of the $\mathrm{S}$ proteins from SARs2, Delta, and Omicro were mapped using the local version of the IBS tool (Fig. 3).

As illustrated in Figure 3, the S proteins of SARs2, Delta, and Omicro include Hemocyanin C domains in both $\mathrm{S} 1$ and S2. As shown in Figure 3, the S protein of SARs2 and Omicro contains the Hemocyanin_N domain. S1 includes the Hemocyanin_N domain of Delta's S protein. As illustrated in Figure 3, the S proteins of SARs2, Delta, and Omicro include Hemocyanin_M domains in both S1 and S2. However, the three viruses had dramatically varied distributions of Tyrosinase_Cu-bd domains. The S protein of Omicro contains only two Tyrosinase_Cu-bd domains. Omicro S Tyrosinase_Cu-bd B is positioned within the Hemocyanin_M domain, but Omicro S Tyrosinase_Cu-bd A is not. SARs2 contains the Tyrosinase_Cu-bd domains entirely within the N-terminal Hemocyanin_M domain. Delta has the Tyrosinase_Cu-bd domains in C-and N-terminal Hemocyanin_M domains. 
Table 11. Hemocyanin activity-related domains of S protein of Delta and Omicron mutants

\begin{tabular}{|c|c|c|c|c|c|}
\hline Protein & Domain & $\begin{array}{c}\text { Alia } \\
\text { s }\end{array}$ & Motif & Start & End \\
\hline \multirow[t]{14}{*}{ Delta $S$} & Hemocyanin_C & A & CEFQFCNDPFLGVYYHKNNKSWME & 118 & 141 \\
\hline & & $\mathrm{B}$ & RVDFCGKGYHLMS & 1026 & 1038 \\
\hline & & $\mathrm{C}$ & AICHDGKAHFPREGV & 1067 & 1081 \\
\hline & Hemocyanin_M & A & WFHAIH & 51 & 56 \\
\hline & & $\mathrm{B}$ & $\begin{array}{l}\text { VYYHKNNKSWMESEFRVYSSANNCTFEYVS } \\
\text { QPFLMD }\end{array}$ & 130 & 165 \\
\hline & & $\mathrm{C}$ & CTMYICGDSTEC & 725 & 736 \\
\hline & & $\mathrm{D}$ & FAMQMAYRF & 885 & 893 \\
\hline & & $\mathrm{E}$ & CGKGYHLM & 1030 & 1037 \\
\hline & & $\mathrm{F}$ & PAQEKNFTTAPAICHDGKAHFPREGVFVSNG & 1056 & 1090 \\
\hline & & & THWF & & \\
\hline & Hemocyanin_N & A & WFHAIH & 51 & 56 \\
\hline & Tyrosinase_Cu-bd & A & WFHAIHV & 51 & 57 \\
\hline & & $\mathrm{B}$ & IKVCEFQFCNDPFLGVYYHKNNKSWME & 115 & 141 \\
\hline & & $\mathrm{C}$ & PAICHDGKAHFPREG & 1066 & 1080 \\
\hline \multirow[t]{11}{*}{ Omicro S } & Hemocyanin_C & A & HKNNKSWM & 141 & 148 \\
\hline & & $\mathrm{B}$ & RVDFCGKGYHLMS & 1036 & 1048 \\
\hline & & $\mathrm{C}$ & ICHDGKAH & 1078 & 1085 \\
\hline & & $\mathrm{D}$ & $\begin{array}{l}\text { RKDGEWVLLSTFLGRSLEVLFQGPGHHHHHH } \\
\text { HHSAWSHPQFE }\end{array}$ & 1222 & 1263 \\
\hline & Hemocyanin_M & A & CEFQFCNDPFLDHKNNKSWMESE & 129 & 151 \\
\hline & & $\mathrm{B}$ & MTKTSVDCTMYICGDSTEC & 728 & 746 \\
\hline & & $\mathrm{C}$ & WTFGAGPALQIPFPMQMAYR & 883 & 902 \\
\hline & & $\mathrm{D}$ & $\begin{array}{l}\text { TFLGRSLEVLFQGPGHHHHHHHHSAWSHPQF } \\
\text { EKGGG }\end{array}$ & 1232 & 1267 \\
\hline & Hemocyanin_N & A & FQGPGHHHHHHHHSAWSHPQFEK & 1242 & 1264 \\
\hline & Tyrosinase_Cu-bd & A & PAICHDGKAHFPREG & 1076 & 1090 \\
\hline & & $\mathrm{B}$ & GPGHHHHHHHHSAWSHPQFEKG & 1244 & 1265 \\
\hline
\end{tabular}

The above distributions suggest that the S of SARs2 S, the Delta $\mathrm{S}$ variant, represents the cell agglutination and immune interference activity of the entire sequence. However, since Omicro's $\mathrm{S}$ lacks the tyrosinase_Cu-bd domain, cell agglutination and phenoloxidase activity cannot be fully achieved. On the $\mathrm{S}$ sequence, the Delta variant exhibits phenoloxidase activity. The copper-binding site of Delta $\mathrm{S}$ is highly exposed and readily possesses phenoloxidase activity. SARs2 $\mathrm{S}$ has a copper-binding site in the membrane fusion domain, which requires full exposure of SARs S Tyrosinase_Cu-bd A-C and binding to copper to function as a phenoloxidase. Delta S, and Omicro $\mathrm{S}$ all lack or lose their phenoloxidase activity. Since SARs2 S, Delta S, and Omicro S all have tyrosinase $\mathrm{Cu}$ binding sites, all three $\mathrm{S}$ proteins act as oxygen carriers. Due to the easily accessible copper binding site of Delta S1, Delta S has a high oxygen transport capacity. This may explain why Delta patients have higher viral loads and lower levels of respiratory distress than SARs2 patients. Omicro S developed into a hemocyanin-like protein capable of carrying oxygen molecules but lacking immunological agglutination and phenoloxidase functions. This may 
explain why Omicro patients experience fewer severe symptoms and no apparent respiratory distress.

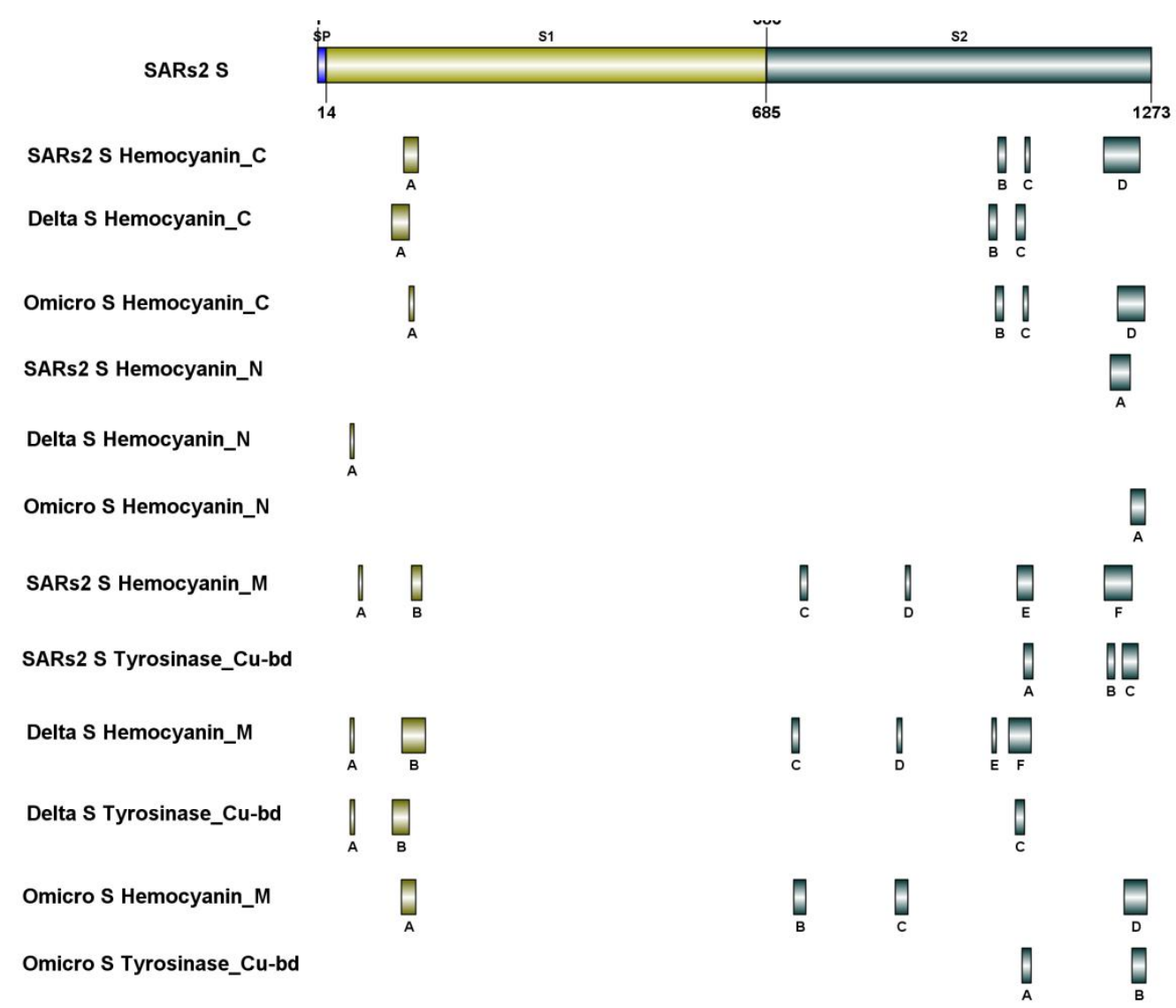

Figure 3. Comparison of hemocyanin activity-related regions of SARs2, Delta, and Omicro S proteins. IBS tools are used to create the diagram(92).

ORF3a mutations had no effect on oxygen supply with reduced hemoglobin. We drew the ORF3a's mutation sites of the hemoglobin attacked-associated domain for SARs2, Delta, and Omicro using a local version of the IBS program (Figure 4). The haeme_bd, NEAT, peptidase S6, IsdA, IsdC and IsdH, ABM, and HemS domains of the SARs2 ORF3a protein are all located within the heme-binding "CWKCR" or "CYDYC" motif, as shown in Figure 4. It suggests that these domains are involved in the degradation, capture, and breakdown of hemoglobin.

Omicro ORF3a does not contain mutations. Delta ORF3a contains two single site mutation 26 and 275. Their mutations are S->L and L->F. Notably, the two Delta ORF3a mutation sites are not located in the Haem_bd, NEAT, peptidase S6, IsdA, IsdC, and IsdH, ABM or HemS domains described above. Therefore, these two point mutations are unlikely to affect ORF3a's hemoglobin attacked, heme capture, or degrade functions. 
SARs2 ORF3a

SARs2 ORF3a Haem_bd

SARs2 ORF3a NEAT

SARs2 ORF3a Peptidase $\mathbf{6} 6$

SARs2 ORF3a IsdA

SARs2 ORF3a IsdC

SARs2 ORF3a IsdH

SARs2 ORF3a ABM

SARs2 ORF3a Hems
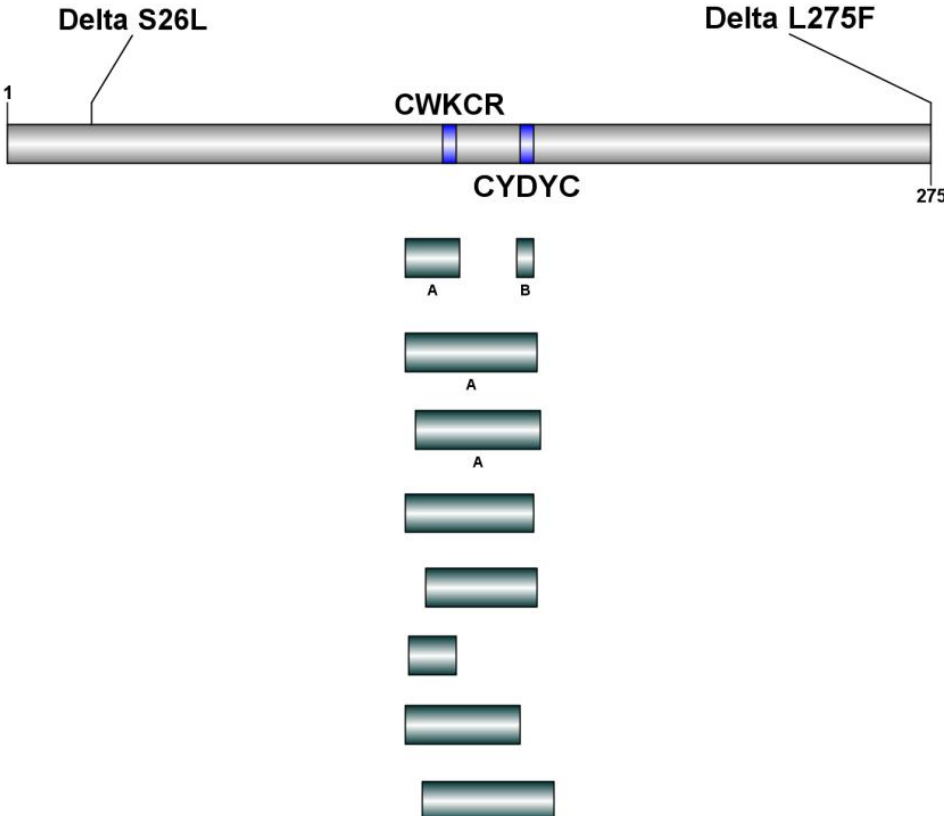

Figure 4. Hemoglobin degradation, heme capture, and disassembly related domains of ORF3a proteins of SARs2, Delta, and Omicro. The figure is drawn with the IBS tool(92).

\section{Discussion}

\section{1 $\mathrm{S}$ and $\mathrm{E}$ protein bind porphyrin to cause high viral infection}

The furtherevolution of the novel coronavirus also displays some paradoxical characteristics. The current theory reveals that the novel coronavirus binds to the human ACE2 receptor through a spike protein. The novel coronavirus enters human cells in the form of phagocytosis. The novel coronavirus pneumonia is highly contagious. What causes the high infectivity of the novel coronavirus? The structural proteins $\mathrm{S}, \mathrm{M}, \mathrm{E}$, and $\mathrm{N}$ have the production and binding domains of porphyrin, according to the research.We believe that in addition to the invasive method of spike-ACE2, it should maintain the original invasive pattern. Medical workers have detected the novel coronavirus from urine, saliva, feces, and blood sincethe virus can live in body fluids. In such media, the porphyrin is a prevalent substance, and porphyrin compounds are a class of nitrogen-containing polymers. Existing studies have shown that they have a strong ability to locate and penetrate cell membranes. Therefore, the novel coronavirus may also directly penetrate the human cell membrane through linking porphyrin.

\subsection{Higher hemoglobin causes higher morbidity}

The novel coronavirus pneumonia might be closely related to abnormal hemoglobin metabolism in humans. The number of hemoglobin is a significant blood biochemical indicator, and the content varies with genders. The number of normal men is significantly higher than that of normal women, which might also be a reason why men are more likely to be infected with the novel coronavirus pneumonia than women. Besides, most patients with the novel coronavirus 
pneumonia are themiddle-aged and older adults, whilemany of these patients have underlying diseases such as diabetes. Diabetic patients have higher glycated hemoglobin which is deoxyhemoglobin and is also a combination of hemoglobin and blood glucose, which is another reason for the high infection rate for the elder people. This present study has confirmed that ORF3a could coordinately attack the heme on the beta chain of hemoglobin. Both oxygenated hemoglobin and deoxygenated hemoglobin are attacked, but the latter is more attacked by the virus. During the attack, the positions of ORF3a are slightly different, which shows that the higher the hemoglobin content, the higher the risk of disease. However, it is not sure that the disease rate incited by abnormal hemoglobin (structural) is relatively low. The hemoglobin of patients and rehabilitees could be detected for further research and treatment.

\subsection{Interfering with the normal heme anabolic pathway}

This article held that the virus directly interfered with the assembly of human hemoglobin. The main reason was that the normal heme was too low. Heme joins in critical biological activities such as regulation of gene expression and protein translation, and the porphyrin is an essential material for the synthesis of the heme. As the existing traces show there is too much free iron in the body of critically ill patients, it could be that the virus-producing molecule competes with iron for the porphyrin, inhibiting the heme anabolic pathway and causing symptoms in humans. It is not clear whether the spatial molecular structure of the heme and porphyrins in patients with porphyria is the same as that in healthy people. If there is an abnormal structure, it is unobvious whether this porphyrin can bind to a viral protein to form a complex, or whether a viral protein can attack this heme. It could be proved by clinical and experimental research.

\subsection{Novel coronavirus has a strong carcinogenicity}

Numerous published studies demonstrate that after cancer patients get COVID-19, their overall risk of cancer deterioration increases. Numerous clinical studies have established that COVID-19 patients exhibit symptoms consistent with oxidative stress damage. Under conditions of severe oxidative stress, the patient's ROS control mechanism becomes disorganized. The dysregulation of reactive oxygen species (ROS) is a critical element in carcinogenesis. The lungs of the deceased from COVID-19 were mucus-filled, and the deteriorated lung tissue resembled adenocarcinoma-like alterations. We discovered that proteins such as $\mathrm{S}$ have a melanoma domain during our search for antigen and membrane fusion domains. Many malignancies, including lung cancer, have pathogenic proteins that contain melanoma domains. Besides, we were conducting computer research and discovered that proteins such as S contain p53 domains. Normal cells could develop cancer cells when the P53 protein was mutated. We believe that the SARS-COV-2 virus is highly carcinogenic based on these comprehensive characteristics.

\section{Conclusion}

Investigating the factors underlying decreased respiratory function in COVID-19 patients may aid in the saving of many lives. The conserved domain search method was used to investigate the biological roles of certain SARs-COV-2 proteins. The findings indicate that the SARS-COV-2 virus proteins contains regions capable of binding porphyrin and synthesizing heme. $\mathrm{S}$ and ORF3a have the ability to bind to hemoglobin. ORF3a's Arg134 and E's Cys44 have heme-iron binding sites, respectively. ORF3a contains Haem bd, NEAT (IsdA, IsdC, and IsdH), Peptidase S6, 
ABM, and HemS domains. All are found across the "CWKCR" heme-binding site. It indicates that the ORF3a protein can bind to the hemoglobin 1-beta chain and collect and degrade heme into iron and porphyrin. The S protein exhibits hemocyanin-like activity and can transport oxygen molecules via copper binding. The $\mathrm{S}$ is triggered by binding to copper oxygen, producing melanin and activating the human immune response through phenoloxidase domains. In patients with COVID-19, the ORF3a protein's action to attack hemoglobin and degrade heme caused respiratory tissues and organs damage. It resulted in a range of respiratory distress and coagulation symptoms in patients, as well as a disruption of normal heme metabolism. However, the oxygen-carrying activity of the S protein's hemocyanin-like domain enhanced the patient's respiration function. The $S$ protein's phenoloxidase function was decreased or missing in the Delta and Omicron viriant, resulting in a weaker immunological response and greater immune escape. However, the ability of ORF3a to target hemoglobin was not diminished. The mutant virus utilized the S protein's high-efficiency oxygen transportability to reduce respiratory distress symptoms in patients.

\section{Declarations}

\section{Ethics approval and consent to participate}

Not applicable.

\section{Consent for publication}

Not applicable.

\section{Availability of data and material}

The datasets and results supporting the conclusions of this article are available at https://pan.baidu.com/s/1GZcnrCBQN5XufMcTNDXjaA, code: jcjs. Or: https://mega.nz/folder/47AVRQrb\#h0EiMUehhIvj8bY8U6pleg

\section{Competing interests}

The authors declare that they have no competing interests.

\section{Funding}

This work was funded by a grant from the Zigong City Key Science and Technology Plan Project (award number: 2021YLSF27, grant recipient: LWZ). This work was partly funded by a grant from the Talent Introduction Project of Sichuan University of Science and Engineering (award number: 2018RCL20, grant recipient: WZL).

\section{Author's contribution}

Funding was obtained by WZL. Besides, design, analysis and writing are finished by WZL, while data curation and manuscript check are undertaken by HLL. Both authors have read and agreed to the published version of the manuscript.

\section{Acknowledgements}

Not applicable.

\section{Author details}


${ }^{1}$ School of Computer Science and Engineering, Sichuan University of Science \& Engineering, Zigong, 643002, China.

${ }^{2}$ School of Life Science and Food Engineering, Yibin University, Yibin, 644000, China.

\section{References}

1. Liang, W., Z. Feng, S. Rao, C. Xiao, X. Xue, Z. Lin, Q. Zhang, and W. Qi. 2020. Diarrhoea may be underestimated: a missing link in 2019 novel coronavirus. Gut 69: 1141-1143.

2. Chan, J. F.-W., S. Yuan, K.-H. Kok, K. K.-W. To, H. Chu, J. Yang, F. Xing, J. Liu, C. C.-Y. Yip, and R. W.-S. Poon. 2020. A familial cluster of pneumonia associated with the 2019 novel coronavirus indicating person-to-person transmission: a study of a family cluster. The lancet 395 : 514-523.

3. Hassan, S. A., F. N. Sheikh, S. Jamal, J. K. Ezeh, and A. Akhtar. 2020. Coronavirus (COVID-19): a review of clinical features, diagnosis, and treatment. Cureus 12.

4. Lippi, G., A. M. South, and B. M. Henry. 2020. Electrolyte imbalances in patients with severe coronavirus disease 2019 (COVID-19). Annals of clinical biochemistry 57: 262-265.

5. Wu, Y., X. Xu, Z. Chen, J. Duan, K. Hashimoto, L. Yang, C. Liu, and C. Yang. 2020. Nervous system involvement after infection with COVID-19 and other coronaviruses. Brain, behavior, and immunity 87: 18-22.

6. Mungroo, M. R., N. A. Khan, and R. Siddiqui. 2020. Novel coronavirus: current understanding of clinical features, diagnosis, pathogenesis, and treatment options. Pathogens 9: 297.

7. Lu, L., W. Xiong, D. Liu, J. Liu, D. Yang, N. Li, J. Mu, J. Guo, W. Li, and G. Wang. 2020. New onset acute symptomatic seizure and risk factors in coronavirus disease 2019: a retrospective multicenter study. Epilepsia 61: e49-e53.

8. Wang, H.-Y., X.-L. Li, Z.-R. Yan, X.-P. Sun, J. Han, and B.-W. Zhang. 2020. Potential neurological symptoms of COVID-19. Therapeutic advances in neurological disorders 13: 1756286420917830.

9. Moriguchi, T., N. Harii, J. Goto, D. Harada, H. Sugawara, J. Takamino, M. Ueno, H. Sakata, K. Kondo, and N. Myose. 2020. A first case of meningitis/encephalitis associated with SARS-Coronavirus-2. International journal of infectious diseases 94: 55-58.

10. Liu, K., M. Pan, Z. Xiao, and X. Xu. 2020. Neurological manifestations of the coronavirus (SARS-CoV-2) pandemic 2019-2020. Journal of Neurology, Neurosurgery \& Psychiatry 91: 669-670.

11. Xiong, T.-Y., S. Redwood, B. Prendergast, and M. Chen. 2020. Coronaviruses and the cardiovascular system: acute and long-term implications. European heart journal.

12. Zhang, C., L. Shi, and F.-S. Wang. 2020. Liver injury in COVID-19: management and challenges. The lancet Gastroenterology \& hepatology 5: 428-430.

13. Naicker, S., C.-W. Yang, S.-J. Hwang, B.-C. Liu, J.-H. Chen, and V. Jha. 2020. The novel coronavirus 2019 epidemic and kidneys. Kidney International 97: 824-828.

14. Pan, F., T. Ye, P. Sun, S. Gui, B. Liang, L. Li, D. Zheng, J. Wang, R. L. Hesketh, and L. Yang. 2020. Time course of lung changes on chest CT during recovery from 2019 novel coronavirus (COVID-19) pneumonia. Radiology.

15. Poggiali, E., A. Dacrema, D. Bastoni, V. Tinelli, E. Demichele, P. Mateo Ramos, T. Marcianò, M. Silva, A. Vercelli, and A. Magnacavallo. 2020. Can lung US help critical care clinicians in the early diagnosis of novel coronavirus (COVID-19) pneumonia? Radiology 295: E6-E6. 
16. Hong, X., J. Xiong, Z. Feng, and Y. Shi. 2020. Extracorporeal membrane oxygenation (ECMO): does it have a role in the treatment of severe COVID-19? International Journal of Infectious Diseases 94: 78-80.

17. Namendys-Silva, S. A. 2020. ECMO for ARDS due to COVID-19. Heart \& Lung: The Journal of Cardiopulmonary and Acute Care 49: 348-349.

18. Henry, B. M., and G. Lippi. 2020. Poor survival with extracorporeal membrane oxygenation in acute respiratory distress syndrome (ARDS) due to coronavirus disease 2019 (COVID-19): pooled analysis of early reports. Journal of critical care 58: 27.

19. Lu, C., and N. Hou. 2020. Skin Hyperpigmentation in Coronavirus Disease 2019 Patients: Is Polymyxin B the Culprit? Frontiers in Pharmacology 11.

20. Genovese, G., C. Moltrasio, E. Berti, and A. V. Marzano. 2021. Skin manifestations associated with COVID-19: current knowledge and future perspectives. Dermatology: 1-12.

21. Recalcati, S. 2020. Cutaneous manifestations in COVID-19: a first perspective. J Eur Acad Dermatol Venereol 34.

22. Olguín-Manríquez, F., L. Cernichiaro-Espinosa, A. Olguín-Manríquez, R. Manríquez-Arias, E. O. Flores-Villalobos, and P. A. Kawakami-Campos. 2021. Unilateral acute posterior multifocal placoid pigment epitheliopathy in a convalescent COVID-19 patient. International journal of retina and vitreous $7: 41$.

23. Yagci, B. A., F. Atas, M. Kaya, and G. Arikan. 2021. COVID-19 Associated Bilateral Acute Iris Transillumination. Ocular immunology and inflammation 29: 719-721.

24. Iozumi, K., G. E. Hoganson, R. Pennella, M. A. Everett, and B. B. Fuller. 1993. Role of tyrosinase as the determinant of pigmentation in cultured human melanocytes. Journal of Investigative Dermatology 100: 806-811.

25. Chandran, R., L. Feller, J. Lemmer, and R. A. G. Khammissa. 2016. HIV-Associated Oral Mucosal Melanin Hyperpigmentation: A Clinical Study in a South African Population Sample. AIDS Research and Treatment 2016: 8389214.

26. Sidiropoulou, P., A. O. Docea, V. Nikolaou, M.-S. Katsarou, D. A. Spandidos, A. Tsatsakis, D. Calina, and N. Drakoulis. 2021. Unraveling the roles of vitamin D status and melanin during Covid-19 (Review). Int J Mol Med 47: 92-100.

27. Nosanchuk, J. D., and A. Casadevall. 2006. Impact of melanin on microbial virulence and clinical resistance to antimicrobial compounds. Antimicrob Agents Chemother 50: 3519-3528.

28. Wang, Y., P. Aisen, and A. Casadevall. 1995. Cryptococcus neoformans melanin and virulence: mechanism of action. Infection and immunity 63: 3131-3136.

29. Zughaier, S. M., H. C. Ryley, and S. K. Jackson. 1999. A melanin pigment purified from an epidemic strain of Burkholderia cepacia attenuates monocyte respiratory burst activity by scavenging superoxide anion. Infection and immunity 67: 908-913.

30. Adachi, K., T. Hirata, K. Nagai, and M. Sakaguchi. 2001. Hemocyanin a most likely inducer of black spots in kuruma prawn Penaeus japonicus during storage. Journal of Food Science 66: 1130-1136.

31. García-Carreño, F. L., K. Cota, and M. A. Navarrete Del Toro. 2008. Phenoloxidase activity of hemocyanin in whiteleg shrimp Penaeus vannamei: conversion, characterization of catalytic properties, and role in postmortem melanosis. Journal of Agricultural and Food Chemistry 56: 6454-6459. 
32. Coates, C. J., and J. Nairn. 2013. Hemocyanin-derived phenoloxidase activity: a contributing factor to hyperpigmentation in Nephrops norvegicus. Food chemistry 140: 361-369.

33. Decker, H., and F. Tuczek. 2000. Tyrosinase/catecholoxidase activity of hemocyanins: structural basis and molecular mechanism. Trends in biochemical sciences 25: 392-397.

34. Cerenius, L., and K. Söderhäll. 2004. The prophenoloxidase-activating system in invertebrates. Immunological reviews 198: 116-126.

35. Martínez-Alvarez, O., C. Gómez-Guillén, and P. Montero. 2008. Presence of hemocyanin with diphenoloxidase activity in deepwater pink shrimp (Parapenaeus longirostris) post mortem. Food chemistry 107: 1450-1460.

36. Lu, A., Q. Zhang, J. Zhang, B. Yang, K. Wu, W. Xie, Y.-X. Luan, and E. Ling. 2014. Insect prophenoloxidase: the view beyond immunity. Frontiers in Physiology 5.

37. Markl, J., and H. Decker. 1992. Molecular structure of the arthropod hemocyanins. In Blood and tissue oxygen carriers. Springer. 325-376.

38. Linzen, B., N. Soeter, A. Riggs, H.-J. Schneider, W. Schartau, M. Moore, E. Yokota, P. Behrens, H. Nakashima, and T. Takagi. 1985. The structure of arthropod hemocyanins. Science 229: 519-524.

39. Zhao, X., L. Guo, Y. Zhang, Y. Liu, X. Zhang, J. Lun, J. Chen, and Y. Li. 2012. SNPs of hemocyanin C-terminal fragment in shrimp Litopenaeus vannamei. FEBS letters 586: 403-410.

40. Qin, Z., V. S. Babu, Q. Wan, A. Muhammad, J. Li, J. Lan, and L. Lin. 2018. Antibacterial activity of hemocyanin from red swamp crayfish (Procambarus clarkii). Fish \& shellfish immunology 75 : 391-399.

41. Coates, C. J., and H. Decker. 2017. Immunological properties of oxygen-transport proteins: hemoglobin, hemocyanin and hemerythrin. Cellular and Molecular Life Sciences 74: 293-317.

42. Magnus, K. A., B. Hazes, H. Ton-That, C. Bonaventura, J. Bonaventura, and W. G. Hol. 1994. Crystallographic analysis of oxygenated and deoxygenated states of arthropod hemocyanin shows unusual differences. Proteins: Structure, Function, and Bioinformatics 19: 302-309.

43. Lutz, D. 2010. The Many Colors of. chemmatters: 5.

44. Bux, K., S. A. Ali, and S. T. Moin. 2018. Hydration facilitates oxygenation of hemocyanin: perspectives from molecular dynamics simulations. European Biophysics Journal 47: 925-938.

45. Wawrowski, A., P. G. Matthews, E. Gleixner, L. Kiger, M. C. Marden, T. Hankeln, and T. Burmester. 2012. Characterization of the hemoglobin of the backswimmer Anisops deanei (Hemiptera). Insect biochemistry and molecular biology 42: 603-609.

46. Terwilliger, N. B. 2015. Oxygen transport proteins in Crustacea: hemocyanin and hemoglobin. Physiology 4: 359-390.

47. Pick, C., S. Hagner-Holler, and T. Burmester. 2008. Molecular characterization of hemocyanin and hexamerin from the firebrat Thermobia domestica (Zygentoma). Insect biochemistry and molecular biology 38: 977-983.

48. Flachsbarth, S., M. Kruse, and T. Burmester. 2017. Distribution and hypoxia-regulation of haemocyanin in springtails (Collembola). Insect molecular biology 26: 633-641.

49. Chen, B., R. Ma, D. Ding, L. Wei, and L. Kang. 2017. Aerobic respiration by haemocyanin in the embryo of the migratory locust. Insect molecular biology 26: 461-468.

50. Lehtonen, M. P., and L. E. Burnett. 2016. Effects of hypoxia and hypercapnic hypoxia on oxygen transport and acid-base status in the Atlantic blue crab, Callinectes sapidus, during exercise. Journal of Experimental Zoology Part A: Ecological Genetics and Physiology 325: 598-609. 
51. Head, J. 2010. The effects of hypoxia on hemocyanin regulation in Cancer magister: Possible role of Hypoxia-Inducible Factor-1. Journal of experimental marine biology and ecology 386: 77-85.

52. McMahon, B. R. 2001. Respiratory and circulatory compensation to hypoxia in crustaceans. Respiration physiology 128: 349-364.

53. Millet, G. P., and T. Debevec. 2021. Altitude and COVID-19: Friend or foe? A narrative review. 8: e14615.

54. Kobayashi, M., K. Kitayama, M. Watanabe, and N. Makino. 1995. The oxygen transport efficiency of arthropod hemocyanins. Zoological science 12: 271-276.

55. Higgins, C. 2014. Oxygen saturation-better measured than calculated.

56. Otto, B. R., R. Sijbrandi, J. Luirink, B. Oudega, J. G. Heddle, K. Mizutani, S. Y. Park, and J. R. Tame. 2005. Crystal structure of hemoglobin protease, a heme binding autotransporter protein from pathogenic Escherichia coli. The Journal of biological chemistry 280: 17339-17345.

57. Mitra, A., D. M. Dwyre, M. Schivo, G. R. Thompson III, S. H. Cohen, N. Ku, and J. P. Graff. 2020. Leukoerythroblastic reaction in a patient with COVID-19 infection. American journal of hematology.

58. Bhardwaj, K., P. Liu, J. L. Leibowitz, and C. C. Kao. 2012. The coronavirus endoribonuclease Nsp15 interacts with retinoblastoma tumor suppressor protein. Journal of virology 86: 4294-4304.

59. Emmons, R., L. Oshiro, H. Johnson, and E. Lennette. 1972. Intra-erythrocytic location of Colorado tick fever virus. Journal of General Virology 17: 185-195.

60. Szempruch, A. J., S. E. Sykes, R. Kieft, L. Dennison, A. C. Becker, A. Gartrell, W. J. Martin, E. S. Nakayasu, I. C. Almeida, and S. L. Hajduk. 2016. Extracellular vesicles from Trypanosoma brucei mediate virulence factor transfer and cause host anemia. Cell 164: 246-257.

61. Howell, S. A., I. Well, S. L. Fleck, C. Kettleborough, C. R. Collins, and M. J. Blackman. 2003. A single malaria merozoite serine protease mediates shedding of multiple surface proteins by juxtamembrane cleavage. Journal of Biological Chemistry 278: 23890-23898.

62. Otto, B. R., S. J. van Dooren, J. H. Nuijens, J. Luirink, and B. Oudega. 1998. Characterization of a hemoglobin protease secreted by the pathogenic Escherichia coli strain EB1. The Journal of experimental medicine 188: 1091-1103.

63. Sabotič, J., and J. Kos. 2012. Microbial and fungal protease inhibitors - current and potential applications. Applied Microbiology and Biotechnology 93: 1351-1375.

64. Zhu, H., G. Xie, M. Liu, J. S. Olson, M. Fabian, D. M. Dooley, and B. Lei. 2008. Pathway for heme uptake from human methemoglobin by the iron-regulated surface determinants system of Staphylococcus aureus. Journal of biological chemistry 283: 18450-18460.

65. Pilpa, R. M., S. A. Robson, V. A. Villareal, M. L. Wong, M. Phillips, and R. T. Clubb. 2009. Functionally distinct NEAT (NEAr Transporter) domains within the Staphylococcus aureus IsdH/HarA protein extract heme from methemoglobin. Journal of Biological Chemistry 284: 1166-1176.

66. Krishna Kumar, K., D. A. Jacques, G. Pishchany, T. Caradoc-Davies, T. Spirig, G. R. Malmirchegini, D. B. Langley, C. F. Dickson, J. P. Mackay, R. T. Clubb, E. P. Skaar, J. M. Guss, and D. A. Gell. 2011. Structural Basis for Hemoglobin Capture by $<\mathrm{em}>$ Staphylococcus aureus $</$ em $>$ Cell-surface Protein, IsdH $<$ sup $>$ $</$ sup $>$. Journal of Biological Chemistry 286: 38439-38447. 
67. Krishna Kumar, K., C. F. Dickson, M. J. Weiss, J. P. Mackay, and D. A. Gell. 2010. AHSP ( $\alpha$-haemoglobin-stabilizing protein) stabilizes apo- $\alpha$-haemoglobin in a partially folded state. Biochemical Journal 432: 275-282.

68. Villareal, V. A., R. M. Pilpa, S. A. Robson, E. A. Fadeev, and R. T. Clubb. 2008. The IsdC protein from Staphylococcus aureus uses a flexible binding pocket to capture heme. The Journal of biological chemistry 283: 31591-31600.

69. Sharp, K. H., S. Schneider, A. Cockayne, and M. Paoli. 2007. Crystal structure of the heme-IsdC complex, the central conduit of the Isd iron/heme uptake system in Staphylococcus aureus. Journal of Biological Chemistry 282: 10625-10631.

70. Hurd, A. F., J. Garcia-Lara, Y. Rauter, M. Cartron, R. Mohamed, and S. J. Foster. 2012. The iron-regulated surface proteins IsdA, IsdB, and IsdH are not required for heme iron utilization in Staphylococcus aureus. FEMS Microbiology Letters 329: 93-100.

71. Bottino-Rojas, V., O. A. Talyuli, N. Jupatanakul, S. Sim, G. Dimopoulos, T. M. Venancio, A. C. Bahia, M. H. Sorgine, P. L. Oliveira, and G. O. Paiva-Silva. 2015. Heme signaling impacts global gene expression, immunity and dengue virus infectivity in Aedes aegypti. PLoS One 10: e0135985.

72. Sigala, P. A., and D. E. Goldberg. 2014. The peculiarities and paradoxes of Plasmodium heme metabolism. Annu Rev Microbiol 68: 259-278.

73. Kaluka, D. 2019. Heme-containing proteins in the Malaria Parasite. science-seminar 6: https://pillars.taylor.edu/science-seminar/6.

74. Spring, F. A., C. H. Holmes, K. L. Simpson, W. J. Mawby, M. Jules Mattes, Y. Okubo, and S. F. Parsons. 1997. The Oka blood group antigen is a marker for the M6 leukocyte activation antigen, the human homolog of OX-47 antigen, basigin and neurothelin, an immunoglobulin superfamily molecule that is widely expressed in human cells and tissues. European journal of immunology 27: 891-897.

75. Anzaldi, L. L., and E. P. Skaar. 2010. Overcoming the heme paradox: heme toxicity and tolerance in bacterial pathogens. Infection and immunity 78: 4977-4989.

76. Wilks, A., and M. P. Schmitt. 1998. Expression and Characterization of a Heme Oxygenase (Hmu O) fromCorynebacterium diphtheriae: IRON ACQUISITION REQUIRES OXIDATIVE CLEAVAGE OF THE HEME MACROCYCLE. Journal of Biological Chemistry 273: 837-841.

77. Skaar, E. P., A. H. Gaspar, and O. Schneewind. 2004. IsdG and IsdI, heme-degrading enzymes in the cytoplasm of Staphylococcus aureus. The Journal of biological chemistry 279: 436-443.

78. Létoffé, S., G. Heuck, P. Delepelaire, N. Lange, and C. Wandersman. 2009. Bacteria capture iron from heme by keeping tetrapyrrol skeleton intact. Proceedings of the National Academy of Sciences 106: 11719-11724.

79. Stojiljkovic, I., and K. Hantke. 1994. Transport of haemin across the cytoplasmic membrane through a haemin-specific periplasmic binding-protein-dependent transport system in Yersinia enterocolitica. Molecular microbiology 13: 719-732.

80. Suits, M. D., G. P. Pal, K. Nakatsu, A. Matte, M. Cygler, and Z. Jia. 2005. Identification of an Escherichia coli O157: $\mathrm{H} 7$ heme oxygenase with tandem functional repeats. Proceedings of the National Academy of Sciences 102: 16955-16960.

81. Liu, M., H.-J. Boulouis, and F. Biville. 2012. Heme Degrading Protein HemS Is Involved in Oxidative Stress Response of Bartonella henselae. PLoS One 7: e37630. 
82. Rabiee, M., N. Rabiee, R. Salarian, and G. Rabiee. 2019. Porphyrin-based nanomaterials. In Introduction to Nanomaterials in Medicine. Morgan \& Claypool Publishers. 6-1-6-24.

83. Sułek, A., B. Pucelik, M. Kobielusz, A. Barzowska, and J. M. Dąbrowski. 2020. Photodynamic Inactivation of Bacteria with Porphyrin Derivatives: Effect of Charge, Lipophilicity, ROS Generation, and Cellular Uptake on Their Biological Activity In Vitro. Int J Mol Sci 21: 8716.

84. Tsubone, T. M., W. K. Martins, C. Pavani, H. C. Junqueira, R. Itri, and M. S. Baptista. 2017. Enhanced efficiency of cell death by lysosome-specific photodamage. 7:6734.

85. Kolarova, H., P. Nevrelova, K. Tomankova, P. Kolar, R. Bajgar, and J. Mosinger. 2008. Production of reactive oxygen species after photodynamic therapy by porphyrin sensitizers. General physiology and biophysics 27: 101-105.

86. Nishida, K., T. Tojo, T. Kondo, and M. Yuasa. 2021. Evaluation of the correlation between porphyrin accumulation in cancer cells and functional positions for application as a drug carrier. Scientific Reports 11: 2046.

87. Clichici, S., A. Filip, D. Daicoviciu, R. M. Ion, T. Mocan, C. Tatomir, L. Rogojan, D. Olteanu, and A. Muresan. 2010. The dynamics of reactive oxygen species in photodynamic therapy with tetra sulfophenyl-porphyrin. Acta physiologica Hungarica 97: 41-51.

88. Gomi, H., K. Hatanaka, T. Miura, and I. Matsuo. 1997. Type of Impaired Porphyrin Metabolism Caused by Hepatitis C Virus Is Not Porphyria Cutanea Tarda but Chronic Hepatic Porphyria. Archives of Dermatology 133: 1170-1171.

89. Romeo, G., and E. Y. Levin. 1969. Uroporphyrinogen 3 cosynthetase in human congenital erythropoietic porphyria. Proceedings of the National Academy of Sciences of the United States of America 63: 856-863.

90. Liu, W., and H. Li. 2021. COVID-19: Invades Erythrocytes through Plasmodium Falciparum Antigen and Complement-Like System. ChemRxiv. Preprint.

91. Torjesen, I. 2022. Covid-19: Peak of viral shedding is later with omicron variant, Japanese data suggest. British Medical Journal Publishing Group.

92. Liu, W., Y. Xie, J. Ma, X. Luo, P. Nie, Z. Zuo, U. Lahrmann, Q. Zhao, Y. Zheng, and Y. Zhao. 2015. IBS: an illustrator for the presentation and visualization of biological sequences. Bioinformatics 31: 3359-3361. 